TRANSACTIONS OF THE

AMERICAN MATHEMATICAL SOCIETY

Volume 354, Number 12, Pages 4969-5003

S 0002-9947(02)03080-5

Article electronically published on August 1, 2002

\title{
THICK POINTS FOR INTERSECTIONS OF PLANAR SAMPLE PATHS
}

\author{
AMIR DEMBO, YUVAL PERES, JAY ROSEN, AND OFER ZEITOUNI
}

Abstract. Let $L_{n}^{X}(x)$ denote the number of visits to $x \in \mathbf{Z}^{2}$ of the simple planar random walk $X$, up to step $n$. Let $X^{\prime}$ be another simple planar random walk independent of $X$. We show that for any $0<b<1 /(2 \pi)$, there are $n^{1-2 \pi b+o(1)}$ points $x \in \mathbf{Z}^{2}$ for which $L_{n}^{X}(x) L_{n}^{X^{\prime}}(x) \geq b^{2}(\log n)^{4}$. This is the discrete counterpart of our main result, that for any $a<1$, the Hausdorff dimension of the set of thick intersection points $x$ for which $\limsup \sup _{r \rightarrow 0} \mathcal{I}(x, r) /\left(r^{2}|\log r|^{4}\right)=a^{2}$, is almost surely $2-2 a$. Here $\mathcal{I}(x, r)$ is the projected intersection local time measure of the disc of radius $r$ centered at $x$ for two independent planar Brownian motions run until time 1. The proofs rely on a "multi-scale refinement" of the second moment method. In addition, we also consider analogous problems where we replace one of the Brownian motions by a transient stable process, or replace the disc of radius $r$ centered at $x$ by $x+r K$ for general sets $K$.

\section{INTRODUCTION}

Let $X_{n}$ denote the simple random walk in the plane $\mathbf{Z}^{2}$ with

$$
L_{n}^{X}(x)=\#\left\{i: X_{i}=x, 0 \leq i \leq n\right\},
$$

the number of visits to $x \in \mathbf{Z}^{2}$ during the first $n$ steps of the walk, and

$$
T_{n}^{X}=\max _{x \in \mathbf{Z}^{2}} L_{n}^{X}(x),
$$

its maximal value. In [5, Theorem 1.1] we showed that

$$
\lim _{n \rightarrow \infty} \frac{T_{n}^{X}}{(\log n)^{2}}=\frac{1}{\pi}, \text { a.s. }
$$

resolving a conjecture of Erdös and Taylor. Further, with $|A|$ counting the number of points in the set $A$, we show there that

$$
\lim _{n \rightarrow \infty} \frac{\log \left|\left\{x \in \mathbf{Z}^{2}: L_{n}^{X}(x) \geq a(\log n)^{2}\right\}\right|}{\log n}=1-\pi a, \text { a.s. }
$$

for any fixed $0<a<1 / \pi$.

Received by the editors May 9, 2001 and, in revised form, April 16, 2002.

2000 Mathematics Subject Classification. Primary 60J55; Secondary 60J65, 28A80, 60G50.

Key words and phrases. Thick points, intersection local time, multi-fractal analysis, stable process.

The first author's research was partially supported by NSF grant \#DMS-0072331.

The second author's research was partially supported by NSF grant \#DMS-9803597.

The third author's research was supported, in part, by grants from the NSF and from PSCCUNY.

The research of all authors was supported, in part, by a US-Israel BSF grant. 
It is natural to consider the situation for intersections of two (or more) independent simple random walks (SRW) in $\mathbf{Z}^{2}$. Thus, for two independent simple random walks $X_{n}, X_{n}^{\prime}$ and $x \in \mathbf{Z}^{2}$, define

$$
T_{n}^{X, X^{\prime}}=\max _{x \in \mathbf{Z}^{2}} L_{n}^{X}(x) L_{n}^{X^{\prime}}(x) .
$$

Here is our analogue of (1.1) and (1.2) for two independent random walks.

Theorem 1.1.

$$
\lim _{n \rightarrow \infty} \frac{T_{n}^{X, X^{\prime}}}{(\log n)^{4}}=\frac{1}{4 \pi^{2}}, \text { a.s. }
$$

Further, for $0<b<1 /(2 \pi)$,

$$
\lim _{n \rightarrow \infty} \frac{\log \left|\left\{x \in \mathbf{Z}^{2}: L_{n}^{X}(x) L_{n}^{X^{\prime}}(x) \geq b^{2}(\log n)^{4}\right\}\right|}{\log n}=1-2 \pi b, \text { a.s. }
$$

We now outline a heuristic leading to Theorem 1.1, With high probability the path $\left\{X_{k}: k \leq n\right\}$ is contained in a disc of radius $n^{1 / 2+o(1)}$. The proof of (1.2) suggests that, with probability $n^{-\pi a+o(1)}$, a point $x$ in that disc is visited by the random walk $\left\{X_{k}: k \leq n\right\}$ at least $a(\log n)^{2}$ times, i.e., $L_{n}^{X}(x) \geq a(\log n)^{2}$. Similarly, the probability that $L_{n}^{X^{\prime}}(x) \geq a^{\prime}(\log n)^{2}$ is $n^{-\pi a^{\prime}+o(1)}$. So, choosing $a^{\prime}=b^{2} / a$, one sees that $L_{n}^{X}(x) L_{n}^{X^{\prime}}(x) \geq b^{2}(\log n)^{4}$ with probability $n^{-\pi\left(a+b^{2} / a\right)+o(1)}$ for some $a>0$. Taking the optimal value $a=b$ suggests the upper bound given in (1.4). Since the events $\left\{L_{n}^{X}(x) L_{n}^{X^{\prime}}(x) \geq b^{2}(\log n)^{4}\right\}$ for $x \in \mathbf{Z}^{2}$ are highly dependent, establishing the complementary lower bound requires a "multi-scale refinement" of the second moment method. Such a technique was developed in [5] for the planar Brownian occupation measure

$$
\mu_{\bar{\theta}}^{W}(A):=\int_{0}^{\bar{\theta}} 1_{A}\left(W_{s}\right) d s,
$$

where $A$ is a measurable subset of $\mathbb{R}^{2},\left\{W_{s}, s \geq 0\right\}$ denotes a planar Brownian path and $\bar{\theta}:=\min \left\{s:\left|W_{s}\right|=1\right\}$. Using strong approximations of Brownian motion by random walks, (1.2) is the outcome of a precise study of those points $x$ in whose neighborhood the Brownian motion spends an unusually large amount of time, in all sufficiently small scales. We showed in [5] that

$$
\lim _{\epsilon \rightarrow 0} \sup _{x \in D(0,1)} \frac{\mu_{\bar{\theta}}^{W}(D(x, \epsilon))}{\epsilon^{2}(\log \epsilon)^{2}}=2
$$

where $D(x, r)$ is the open disc in $\mathbb{R}^{2}$ of radius $r$ centered at $x$. Then defining the set of thick points

$$
\text { Thick }_{a}^{W}=\left\{x \in D(0,1): \lim _{\epsilon \rightarrow 0} \frac{\mu_{\bar{\theta}}^{W}(D(x, \epsilon))}{\epsilon^{2}(\log \epsilon)^{2}}=a\right\}
$$

for any $a \in(0,2]$, another outcome of that study, [5, Theorem 1.4], shows that $\operatorname{dim}\left(\right.$ Thick $\left._{a}^{W}\right)=2-a$, with dim denoting the Hausdorff dimension of a set.

Our goal in this paper is to better understand the essential reason for such a result. We do so by extending it in three different directions:

- considering occupation measure of sets without radial symmetry;

- considering the projected intersection local time for two (or more) independent planar Brownian motions; 
- considering also the projected intersection local time between the recurrent Brownian path and the sample path of a transient (stable) process with jumps.

Turning to the first of these directions, let $K \subseteq D(0,1)$ have area (i.e., Lebesgue measure) $|K|$, and let $K(x, r)=x+r K$. We next show how the results of [5] for thick points are modified when we replace the discs $D(x, \epsilon)$ by $K(x, \epsilon)$. The analogous modification of our study concerning thick points for intersections will then be straightforward and is left to the reader.

Theorem 1.2. If $|\partial K|=0$, then

$$
\lim _{\varepsilon \rightarrow 0} \sup _{x \in \mathbb{R}^{2}} \frac{\mu_{\bar{\theta}}^{W}(K(x, \varepsilon))}{\varepsilon^{2}\left(\log \frac{1}{\varepsilon}\right)^{2}}=\sup _{|x|<1} \limsup _{\varepsilon \rightarrow 0} \frac{\mu_{\bar{\theta}}^{W}(K(x, \varepsilon))}{\varepsilon^{2}\left(\log \frac{1}{\varepsilon}\right)^{2}}=2|K| / \pi, \quad \text { a.s. }
$$

and for any $a \leq 2|K| / \pi$,

$$
\operatorname{dim}\left\{x \in \mathbb{R}^{2}: \lim _{\varepsilon \rightarrow 0} \frac{\mu_{\bar{\theta}}^{W}(K(x, \varepsilon))}{\varepsilon^{2}\left(\log \frac{1}{\varepsilon}\right)^{2}}=a\right\}=2-a \pi /|K|, \quad \text { a.s. }
$$

Here one may replace $\bar{\theta}$ by any deterministic $0<T<\infty$. We also note that all remarks following Theorem 1.3 in [5] extend verbatim to the situation where the set $K$ is used instead of $D(0,1)$.

Our main example concerns those points $x$ in whose neighborhood two independent planar Brownian motions have an unusually large "amount" of intersections, in all sufficiently small scales. This is quantified by the projected intersection local time. Let $\left\{W_{s} ; 0 \leq s \leq S\right\}$ and $\left\{W_{t}^{\prime} ; 0 \leq t \leq T\right\}$ be two independent planar Brownian motions started at the origin. For any Borel set $A \subseteq \mathbb{R}^{2}$ we define the projected intersection local time in $A$ by

$$
\mathcal{I}_{S, T}(A)=\lim _{\epsilon \rightarrow 0} \pi \int_{0}^{S} \int_{0}^{T} 1_{A}\left(W_{s}\right) f_{\epsilon}\left(W_{s}-W_{t}^{\prime}\right) d s d t
$$

where the factor $\pi$ is a convenient normalization, and $f_{\epsilon}$ is any approximate $\delta$ function, i.e., we take $f$ to be a nonnegative continuous function supported on the unit disc with $\int f d x=1$ and set $f_{\epsilon}(x)=f(x / \epsilon) / \epsilon^{2}$. It is known that the limit (1.8) exists a.s. and in all $L^{p}$ spaces, and that $\mathcal{I}_{S, T}(\cdot)$ is a measure supported on $\left\{x \in \mathbb{R}^{2} \mid x=W_{s}=W_{t}^{\prime}\right.$ for some $\left.0 \leq s \leq S, 0 \leq t \leq T\right\}$; see [?, Chapter VIII, Theorem 1]. Further, Le Gall [?] shows that, with $\bar{\theta}^{\prime}=\inf \left\{t:\left|W_{t}^{\prime}\right|=1\right\}$, there exists a constant $0<c<\infty$ such that, almost surely, for typical $x$ in the support of $\mathcal{I}_{\bar{\theta}, \bar{\theta}^{\prime}}$,

$$
\limsup _{\varepsilon \rightarrow 0} \frac{\mathcal{I}_{\bar{\theta}, \bar{\theta}^{\prime}}(D(x, \varepsilon))}{\varepsilon^{2}\left(\log \frac{1}{\varepsilon} \log \log \log \frac{1}{\varepsilon}\right)^{2}}=c .
$$

In contrast, our next result describes just how large the projected intersection local time can be in the neighborhood of any exceptional point.

\section{Theorem 1.3.}

$$
\lim _{\varepsilon \rightarrow 0} \sup _{x \in \mathbb{R}^{2}} \frac{\mathcal{I}_{\bar{\theta}, \bar{\theta}^{\prime}}(D(x, \varepsilon))}{\varepsilon^{2}\left(\log \frac{1}{\varepsilon}\right)^{4}}=1, \quad \text { a.s. }
$$

Here also one may replace $\bar{\theta}, \bar{\theta}^{\prime}$ by any deterministic $0<S, T<\infty$.

The next theorem describes the multi-fractal structure of the planar projected intersection local time. 
Theorem 1.4. For any $0<a \leq 1$,

$$
\operatorname{dim}\left\{x \in D(0,1): \lim _{\varepsilon \rightarrow 0} \frac{\mathcal{I}_{\bar{\theta}, \bar{\theta}^{\prime}}(D(x, \varepsilon))}{\varepsilon^{2}\left(\log \frac{1}{\varepsilon}\right)^{4}}=a^{2}\right\}=2-2 a, \quad \text { a.s. }
$$

Also,

$$
\sup _{|x|<1} \limsup _{\varepsilon \rightarrow 0} \frac{\mathcal{I}_{\bar{\theta}, \bar{\theta}^{\prime}}(D(x, \varepsilon))}{\varepsilon^{2}\left(\log \frac{1}{\varepsilon}\right)^{4}}=1, \quad \text { a.s. }
$$

Since $\mathcal{I}_{\bar{\theta}, \bar{\theta}^{\prime}}(D(x, \epsilon))=0$ for any $x \notin\left\{W_{t} \mid 0 \leq t \leq \bar{\theta}\right\}$ and $\epsilon$ small enough, by the uniform dimension doubling property of Brownian motion (see [10] or [17, Eq. $(0.1)]),(1.10)$ is equivalent to

$$
\operatorname{dim}\left\{0 \leq t \leq \bar{\theta}: \lim _{\varepsilon \rightarrow 0} \frac{\mathcal{I}_{\bar{\theta}, \bar{\theta}^{\prime}}\left(D\left(W_{t}, \varepsilon\right)\right)}{\varepsilon^{2}\left(\log \frac{1}{\varepsilon}\right)^{4}}=a^{2}\right\}=1-a, \quad \text { a.s. }
$$

We also have the following analogue of the coarse multi-fractal spectrum:

Proposition 1.5. For all $a<1$,

$$
\lim _{\varepsilon \rightarrow 0} \frac{\log \mathcal{L} e b\left(x: \mathcal{I}_{\bar{\theta}, \bar{\theta}^{\prime}}(D(x, \varepsilon)) \geq a^{2} \varepsilon^{2}(\log \varepsilon)^{4}\right)}{\log \varepsilon}=2 a, \quad \text { a.s. }
$$

Our last example concerns the intersections of planar Brownian paths with other random fractals. Let $\left\{X_{t}\right\}$ denote the symmetric stable process of index $0<\beta<2$ in the plane. As usual, we let

$$
u^{0}(x)=\frac{c_{\beta}}{|x|^{2-\beta}}
$$

denote the 0 -potential density for $\left\{X_{t}\right\}$, where $c_{\beta}=2^{-\beta} \pi^{-1} \Gamma\left(\frac{2-\beta}{2}\right) / \Gamma\left(\frac{\beta}{2}\right)$. Let $\Lambda=\Lambda_{\beta}$ denote the norm of

$$
K_{\beta} f(x)=\int_{D(0,1)} u^{0}(x-y) f(y) d y
$$

considered as an operator from $L^{2}(D(0,1), d x)$ to itself.

For any Borel set $A \subseteq \mathbb{R}^{2}$ we define the projected intersection local time in $A$ for $W$ and $X$ by

$$
\mathcal{I}_{S, T}^{W, X}(A)=\lim _{\epsilon \rightarrow 0} \pi \Lambda^{-1} \int_{0}^{S} \int_{0}^{T} 1_{A}\left(W_{s}\right) f_{\epsilon}\left(W_{s}-X_{t}\right) d s d t
$$

where $f_{\epsilon}$ is any approximate $\delta$-function, and the factor $\pi \Lambda^{-1}$ is a convenient normalization. As was the case for $\mathcal{I}_{S, T}(A)$, it can be shown that the limit (1.15) exists a.s. and in all $L^{p}$ spaces, and that $\mathcal{I}_{S, T}^{W, X}(\cdot)$ is a measure supported on $\left\{x \in \mathbb{R}^{2} \mid x=W_{s}=X_{t}\right.$ for some $\left.0 \leq s \leq S, 0 \leq t \leq T\right\}$. When $T=\infty$ we set $\mathcal{I}_{S}^{W, X}(A):=\mathcal{I}_{S, \infty}^{W, X}(A)$.

We provide next the multi-fractal analysis of thick points for $\mathcal{I}^{W, X}$.

Theorem 1.6. Fix $T \in(0, \infty]$. Then,

$$
\lim _{\varepsilon \rightarrow 0} \sup _{x \in \mathbb{R}^{2}} \frac{\mathcal{I}_{\bar{\theta}, T}^{W, X}(D(x, \varepsilon))}{\varepsilon^{\beta}\left(\log \frac{1}{\varepsilon}\right)^{3}}=\beta^{2} / 4, \quad \text { a.s., }
$$


and for any $0<a \leq \beta / 2$,

$$
\operatorname{dim}\left\{x \in D(0,1): \limsup _{\varepsilon \rightarrow 0} \frac{\mathcal{I}_{\bar{\theta}, T}^{W, X}(D(x, \varepsilon))}{\varepsilon^{\beta}\left(\log \frac{1}{\varepsilon}\right)^{3}}=a^{2}\right\}=\beta-2 a, \quad \text { a.s. }
$$

Equivalently,

$$
\operatorname{dim}\left\{0 \leq t \leq \bar{\theta}: \limsup _{\varepsilon \rightarrow 0} \frac{\mathcal{I}_{\bar{\theta}, T}^{W, X}\left(D\left(W_{t}, \varepsilon\right)\right)}{\varepsilon^{\beta}\left(\log \frac{1}{\varepsilon}\right)^{3}}=a^{2}\right\}=\beta / 2-a, \quad \text { a.s. }
$$

Also,

$$
\sup _{|x|<1} \limsup _{\varepsilon \rightarrow 0} \frac{\mathcal{I}_{\bar{\theta}, T}^{W, X}(D(x, \varepsilon))}{\varepsilon^{\beta}\left(\log \frac{1}{\varepsilon}\right)^{3}}=\beta^{2} / 4, \quad \text { a.s. }
$$

In the next section we estimate the moments of $\mathcal{I}_{\bar{\theta}, \bar{\theta}^{\prime}}(D(x, \varepsilon))$. Applying these estimates we then provide the upper bounds in Theorems 1.3 and 1.4. Section 3 constructs the complementary lower bounds, as well as the lower bounds for Theorem 1.6 dealing with $\mathcal{I}^{W, X}(D(x, \varepsilon))$. Certain key lemmas for deriving the lower bounds are stated and proved in Section 4 . Proposition 1.5 is proved in Section 5. The upper bounds for Theorem 1.6 are derived in Section 6. Section 7 contains the proof of Theorem 1.2 and Section 8 contains that of Theorem 1.1 Complements and open problems are provided in the last section.

\section{INTERSECTION LOCAL TIME ESTIMATES AND UPPER BOUNDS}

Throughout this section, fix $0<r_{1} \leq r$, let $\bar{\theta}_{r}=\inf \left\{s>0:\left|W_{s}\right|=r\right\}$, $\bar{\theta}_{r}^{\prime}=\inf \left\{t>0:\left|W_{t}^{\prime}\right|=r\right\}$ and define

$$
\overline{\mathcal{I}}=\mathcal{I}_{\bar{\theta}_{r}, \bar{\theta}_{r}^{\prime}}\left(D\left(0, r_{1}\right)\right) .
$$

Lemma 2.1. We can find $c<\infty$ such that for all $k \geq 1, r_{1} \leq r / 2 \leq 1$, and $x_{0}, x_{0}^{\prime}$ with $\left|x_{0}\right|=\left|x_{0}^{\prime}\right|=r_{1}$,

$$
\mathbb{E}^{x_{0}, x_{0}^{\prime}}\left(\overline{\mathcal{I}} / r_{1}^{2}\right)^{k} \leq(k !)^{2}\left(\log \left(r / r_{1}\right)+c\right)^{2 k} .
$$

Proof of Lemma 2.1 Let $g_{r}(x, y)$ denote Green's function for $D(0, r)$, i.e., the 0potential density for planar Brownian motion killed when it first hits $D(0, r)^{c}$. It follows from (1.8) (see, e.g., [11, (2.c)]) that

$$
\mathbb{E}^{x_{0}, x_{0}^{\prime}}\left(\overline{\mathcal{I}}^{k}\right)=\pi^{k} \sum_{\sigma, \sigma^{\prime}} \int_{D\left(0, r_{1}\right)^{k}} \prod_{j=1}^{k} g_{r}\left(y_{\sigma(j-1)}, y_{\sigma(j)}\right) g_{r}\left(y_{\sigma^{\prime}(j-1)}, y_{\sigma^{\prime}(j)}\right) d y_{j}
$$

where the sum runs over all pairs of permutations $\sigma, \sigma^{\prime}$ of $\{1, \ldots, k\}$ and we use the convention that $y_{\sigma(0)}=x_{0}, y_{\sigma^{\prime}(0)}=x_{0}^{\prime}$. From [8, page 242] (note that our $g$ is twice theirs), we have

$$
g_{r}(x, y)=\frac{1}{\pi} \log \left(\frac{r}{|x-y|}\left|1-\frac{x \bar{y}}{r^{2}}\right|\right)
$$

where $\bar{y}$ denotes the complex conjugate of $y$. Thus, there exists $c_{o}<\infty$ such that

$$
\frac{1}{\pi} \log \left(\frac{r}{|x-y|}\right)-c_{o} \leq g_{r}(x, y) \leq \frac{1}{\pi} \log \left(\frac{r}{|x-y|}\right)+c_{o}
$$


for all $|x|,|y| \leq r / 2$. Thus, after scaling in $r_{1}$, (2.2) is bounded by

$$
\begin{gathered}
\pi^{k} r_{1}^{2 k} \sum_{\sigma, \sigma^{\prime}} \int_{D(0,1)^{k}} \prod_{j=1}^{k}\left(\frac{1}{\pi} \log \left(r / r_{1}\right)-\frac{1}{\pi} \log \left|y_{\sigma(j)}-y_{\sigma(j-1)}\right|+c_{o}\right) \\
\left(\frac{1}{\pi} \log \left(r / r_{1}\right)-\frac{1}{\pi} \log \left|y_{\sigma^{\prime}(j)}-y_{\sigma^{\prime}(j-1)}\right|+c_{o}\right) d y_{j} \\
\leq r_{1}^{2 k} \sum_{\sigma, \sigma^{\prime}} \sum_{j, j^{\prime}=0}^{k}\left(\log \left(r / r_{1}\right)+c_{o} \pi\right)^{j+j^{\prime}} \sum_{\substack{|\mathcal{A}|=k-j \\
|\mathcal{B}|=k-j^{\prime}}} \prod_{D(0,1)^{k}} \prod_{i \in \mathcal{A}}\left|\log \left(\left|y_{\sigma(i)}-y_{\sigma(i-1)}\right|\right)\right| \prod_{l \in \mathcal{B}}\left|\log \left(\left|y_{\sigma^{\prime}(l)}-y_{\sigma^{\prime}(l-1)}\right|\right)\right| \frac{d y_{1}}{\pi} \cdots \frac{d y_{k}}{\pi}
\end{gathered}
$$

where the last sum goes over all subsets $\mathcal{A}, \mathcal{B}$ of $\{1, \ldots, k\}$ of cardinality $k-j, k-j^{\prime}$, respectively. We then bound the integral in the last line of (2.4) by integrating successively with respect to $d y_{1} \cdots d y_{k}$, using Hölder's inequality and the bound

$$
L:=1 \vee \max _{1 \leq m \leq 4}\left(\sup _{x \in D(0,1)} \int_{D(0,1)}|\log (|x-y|)|^{m} \frac{d y}{\pi}\right)^{1 / m}<\infty
$$

for each integration, noting that the variable $y_{j}$ never appears in more than four factors of $\log (\cdot)$. We thus bound the integral in the last line of (2.4) by $L^{(k-j)+\left(k-j^{\prime}\right)}$ (we use the fact that $L \geq 1$ ), so that (2.4) is bounded by

$$
\begin{aligned}
& r_{1}^{2 k} \sum_{\sigma, \sigma^{\prime}} \sum_{j, j^{\prime}=0}^{k}\left(\log \left(r / r_{1}\right)+c_{o} \pi\right)^{j+j^{\prime}} \sum_{\substack{|\mathcal{A}|=k-j \\
|\mathcal{B}|=k-j^{\prime}}} L^{(k-j)+\left(k-j^{\prime}\right)} \\
& =r_{1}^{2 k}(k !)^{2} \sum_{j, j^{\prime}=0}^{k}\left(\log \left(r / r_{1}\right)+c_{o} \pi\right)^{j+j^{\prime}}\left(\begin{array}{c}
k \\
j
\end{array}\right)\left(\begin{array}{c}
k \\
j^{\prime}
\end{array}\right) L^{(k-j)+\left(k-j^{\prime}\right)} \\
& =r_{1}^{2 k}(k !)^{2}\left(\log \left(r / r_{1}\right)+L+c_{o} \pi\right)^{2 k},
\end{aligned}
$$

and (2.1) then follows.

Lemma 2.2. For any $\delta>0$ we can find $c, y_{0}<\infty$ and $\varepsilon_{0}>0$ so that for all $\varepsilon \leq \varepsilon_{0}$ and $y \geq y_{0}$,

$$
P^{x_{0}, x_{0}^{\prime}}\left(\mathcal{I}_{\bar{\theta}_{2}, \bar{\theta}_{2}^{\prime}}(D(0, \varepsilon)) \geq y^{2} \epsilon^{2}|\log \epsilon|^{2}\right) \leq c \exp (-(1-\delta) 2 y)
$$

for all $x_{0}, x_{0}^{\prime}$ with $\left|x_{0}\right|=\left|x_{0}^{\prime}\right|=\varepsilon$.

Proof of Lemma 2.2. Fix $\delta>0$. By (2.1) with $r=2$ for all $\varepsilon$ sufficiently small and $k$ sufficiently large,

$$
\begin{aligned}
\mathbb{E}^{x_{0}, x_{0}^{\prime}}\left(\mathcal{I}_{\bar{\theta}_{2}, \bar{\theta}_{2}^{\prime}}(D(0, \varepsilon))\right)^{k} & \leq(k !)^{2}(\varepsilon \log (1 / \varepsilon)(1+\delta))^{2 k} \\
& \leq(2 k) !\left(\frac{1}{2} \varepsilon \log (1 / \varepsilon)(1+2 \delta)\right)^{2 k}
\end{aligned}
$$

so that with

$$
F=\sqrt{\frac{\mathcal{I}_{\bar{\theta}_{2}, \bar{\theta}_{2}^{\prime}}(D(0, \varepsilon))}{\frac{1}{4} \varepsilon^{2} \log ^{2}(1 / \varepsilon)(1+2 \delta)^{2}}}
$$


we have for all $k$ large enough,

$$
\mathbb{E}^{x_{0}, x_{0}^{\prime}}\left(F^{2 k}\right) \leq(2 k) !
$$

Hence, $\mathbb{E}^{x_{0}, x_{0}^{\prime}}(\exp (\theta F)) \leq c_{1}$ for some finite $c_{1}=c_{1}(\theta)$ and all $\left|x_{0}\right|=\left|x_{0}^{\prime}\right|=\varepsilon$, $\theta \in[0,1)$. Using this in Chebyscheff's inequality yields

$$
P^{x_{0}, x_{0}^{\prime}}(F \geq y) \leq c e^{-(1-\delta) y}
$$

from which (2.7) follows.

We next provide the required upper bounds in Theorems [1.3 and 1.4. Namely, with the notation

$$
\text { ThickInt }_{\geq a^{2}}=\left\{x \in D(0,1): \limsup _{\varepsilon \rightarrow 0} \frac{\mathcal{I}_{\bar{\theta}, \bar{\theta}^{\prime}}(D(x, \varepsilon))}{\varepsilon^{2}\left(\log \frac{1}{\varepsilon}\right)^{4}} \geq a^{2}\right\},
$$

we will show that for any $a \in(0,1]$,

$$
\operatorname{dim}\left(\text { Thicklnt } \geq a^{2}\right) \leq 2-2 a, \quad \text { a.s. , }
$$

and

$$
\limsup _{\varepsilon \rightarrow 0} \sup _{|x|<1} \frac{\mathcal{I}_{\bar{\theta}, \bar{\theta}^{\prime}}(D(x, \varepsilon))}{\varepsilon^{2}\left(\log \frac{1}{\varepsilon}\right)^{4}} \leq 1, \quad \text { a.s. }
$$

(note that (2.10) provides the upper bound also for (1.11)).

Set $h(\epsilon)=\epsilon^{2}|\log \epsilon|^{4}$ and

$$
z(x, \epsilon):=\mathcal{I}_{\bar{\theta}, \bar{\theta}^{\prime}}(D(x, \varepsilon)) / h(\varepsilon) .
$$

Fix $\delta>0$ small enough $\left(\delta<1 / 22\right.$ will do), and choose a sequence $\tilde{\epsilon}_{n} \downarrow 0$ as $n \rightarrow \infty$ in such a way that $\tilde{\epsilon}_{1}<e^{-2}$ and

$$
h\left(\tilde{\epsilon}_{n+1}\right)=(1-\delta) h\left(\tilde{\epsilon}_{n}\right),
$$

implying that $\tilde{\epsilon}_{n}$ is monotone decreasing in $n$. Since, for $\tilde{\epsilon}_{n+1} \leq \epsilon \leq \tilde{\epsilon}_{n}$ we have

$$
z\left(x, \tilde{\epsilon}_{n}\right)=\frac{h\left(\tilde{\epsilon}_{n+1}\right)}{h\left(\tilde{\epsilon}_{n}\right)} \frac{\mathcal{I}_{\bar{\theta}, \bar{\theta}^{\prime}}\left(D\left(x, \tilde{\epsilon}_{n}\right)\right)}{h\left(\tilde{\epsilon}_{n+1}\right)} \geq(1-\delta) z(x, \epsilon),
$$

it is easy to see that for any $a>0$,

$$
\text { Thicklnt }_{\geq a^{2}} \subseteq D_{a^{2}}:=\left\{x \in D(0,1) \mid \limsup _{n \rightarrow \infty} z\left(x, \tilde{\epsilon}_{n}\right) \geq(1-\delta) a^{2}\right\} .
$$

Let $\left\{x_{j}: j=1, \ldots, \bar{K}_{n}\right\}$ denote a maximal collection of points in $D(0,1)$ such that $\inf _{\ell \neq j}\left|x_{\ell}-x_{j}\right| \geq \delta \tilde{\epsilon}_{n}$. Let $\mathcal{A}_{n}$ be the set of $1 \leq j \leq \bar{K}_{n}$ such that

$$
\mathcal{I}_{\bar{\theta}, \bar{\theta}^{\prime}}\left(D\left(x_{j},(1+\delta) \tilde{\epsilon}_{n}\right)\right) \geq(1-2 \delta) a^{2} h\left(\tilde{\epsilon}_{n}\right) .
$$

Set $\tau_{x, n}=\inf \left\{s: W_{s} \in D\left(x,(1+\delta) \tilde{\epsilon}_{n}\right)\right\}, \tau_{x, n}^{\prime}=\inf \left\{t: W_{t}^{\prime} \in D\left(x,(1+\delta) \tilde{\epsilon}_{n}\right)\right\}$. Applying the strong Markov property and then Lemma 2.2, we have

$$
\begin{aligned}
& \mathbf{P}\left(\mathcal{I}_{\bar{\theta}, \bar{\theta}^{\prime}}\left(D\left(x,(1+\delta) \tilde{\epsilon}_{n}\right)\right) \geq(1-2 \delta) a^{2} h\left(\tilde{\epsilon}_{n}\right)\right) \\
& =\mathbb{E}\left(\tau_{x, n}<\bar{\theta}, \tau_{x, n}^{\prime}<\bar{\theta}^{\prime} ;\right.
\end{aligned}
$$

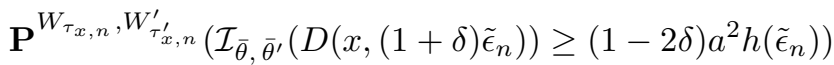

$$
\begin{aligned}
& \leq c \tilde{\epsilon}_{n}^{(1-10 \delta) 2 a}
\end{aligned}
$$

for some $c=c(\delta)<\infty$, all sufficiently large $n$ and any $x \in D(0,1)$. 
Thus, for all sufficiently large $n$, any $j$ and $a>0$,

$$
\mathbf{P}\left(j \in \mathcal{A}_{n}\right) \leq c \tilde{\epsilon}_{n}^{(1-10 \delta) 2 a},
$$

implying that

$$
\mathbb{E}\left|\mathcal{A}_{n}\right| \leq c^{\prime} \tilde{\epsilon}_{n}^{(1-10 \delta) 2 a-2} .
$$

Let $\mathcal{V}_{n, j}=D\left(x_{j}, \delta \tilde{\epsilon}_{n}\right)$. For any $x \in D(0,1)$ there exists $j \in\left\{1, \ldots, \bar{K}_{n}\right\}$ such that $x \in \mathcal{V}_{n, j}$; hence $D\left(x, \tilde{\epsilon}_{n}\right) \subseteq D\left(x_{j},(1+\delta) \tilde{\epsilon}_{n}\right)$. Consequently, $\bigcup_{n \geq m} \bigcup_{j \in \mathcal{A}_{n}} \mathcal{V}_{n, j}$ forms a cover of $D_{a^{2}}$ by sets of maximal diameter $2 \delta \tilde{\epsilon}_{m}$. Fix $a \in(0,2]$. Since $\mathcal{V}_{n, j}$ have diameter $2 \delta \tilde{\epsilon}_{n}$, it follows from (2.14) that for $\gamma=2-(1-11 \delta) 2 a>0$,

$$
\mathbb{E} \sum_{n=m}^{\infty} \sum_{j \in \mathcal{A}_{n}}\left|\mathcal{V}_{n, j}\right|^{\gamma} \leq c^{\prime}(2 \delta)^{\gamma} \sum_{n=m}^{\infty} \tilde{\epsilon}_{n}^{\delta 2 a}<\infty
$$

Thus, $\sum_{n=m}^{\infty} \sum_{j \in \mathcal{A}_{n}}\left|\mathcal{V}_{n, j}\right|^{\gamma}$ is finite a.s., implying that $\operatorname{dim}\left(D_{a^{2}}\right) \leq \gamma$ a.s. Taking $\delta \downarrow 0$ completes the proof of the upper bound (2.9).

Turning to prove (2.10), set $a=(1+\delta) /(1-10 \delta)$ noting that, by (2.15),

$$
\sum_{n=1}^{\infty} \mathbf{P}\left(\left|\mathcal{A}_{n}\right| \geq 1\right) \leq \sum_{n=1}^{\infty} \mathbb{E}\left|\mathcal{A}_{n}\right| \leq c^{\prime} \sum_{n=1}^{\infty} \tilde{\epsilon}_{n}^{2 \delta}<\infty
$$

By Borel-Cantelli, it follows that $\mathcal{A}_{n}$ is empty a.s. for all $n>n_{0}(\omega)$ and some $n_{0}(\omega)<\infty$. By 2.12) we then have

$$
\sup _{\epsilon \leq \tilde{\epsilon}_{n_{0}(\omega)}} \sup _{|x|<1} \frac{\mathcal{I}_{\bar{\theta}, \bar{\theta}^{\prime}}(D(x, \varepsilon))}{\varepsilon^{2}\left(\log \frac{1}{\varepsilon}\right)^{4}} \leq a^{2},
$$

and (2.10) follows by taking $\delta \downarrow 0$.

In proving the lower bound in the following sections we will need a variant of Lemma 2.1 which we now discuss. Let $\rho$ be a fixed measure on $D(0,1)$ with $\int g_{1}(x, y) d \rho(y)$ uniformly bounded and continuous on $D(0,1)$ and let $L_{t}^{\rho}$ denote the continuous additive functional for $\left\{W_{s}\right\}$ with Revuz measure $\rho$. We can define $L_{t}^{\rho}$ as

$$
L_{t}^{\rho}=\lim _{\epsilon \rightarrow 0} \int_{0}^{\bar{\theta}} \int f_{\epsilon}\left(W_{s}-y\right) d \rho(y) d s
$$

where $f_{\epsilon}$ is any approximate identity. Convergence in (2.16) holds a.s., and in all $L^{p}$ spaces. Our interest in such functionals stems from the fact (see [1, Section 2]) that for any $r$,

$$
\mathcal{I}_{\bar{\theta}_{r}, \bar{\theta}_{r}^{\prime}}(A)=L_{\bar{\theta}_{r}}^{\pi \mu_{\bar{\theta}_{r}^{\prime}}^{W^{\prime}} \mid A}
$$

where $\mu_{\bar{\theta}_{r}^{\prime}}^{W^{\prime}}(B)=\int_{0}^{\bar{\theta}_{r}^{\prime}} 1_{B}\left(W_{t}^{\prime}\right) d t$ is the occupation measure with respect to $W^{\prime}$, and for any measure $\nu$ we define the restriction $\nu \mid A(B)=\nu(A \cap B)$. In the sequel, we write $a=(b \pm c) d$ to mean $|a / d-b| \leq c$.

Lemma 2.3. Assume that for some $\gamma_{1}>0, \gamma_{2}>0$ and $c_{1}<\infty$,

$$
\sup _{|x| \leq 2} \frac{\rho(D(x, \varepsilon))}{\varepsilon^{\gamma_{1}}\left(\log \frac{1}{\varepsilon}\right)^{\gamma_{2}}} \leq c_{1}<\infty, \quad \forall \varepsilon>0 .
$$


We can then find $c<\infty$ such that for all $k \geq 1, r_{1} \leq r_{2} \leq r / 2 \leq 1 / 2$, and $x_{0}$ with $\left|x_{0}\right|=r_{2}$, both

$$
\mathbb{E}^{x_{0}}\left(L_{\bar{\theta}_{r}}^{\pi \rho \mid D\left(0, r_{1}\right)}\right)^{k} \leq k !\left(\rho\left(D\left(0, r_{1}\right)\right) \log \left(r / r_{1}\right)+c r_{1}^{\gamma_{1}}\left(\log \left(1 / r_{1}\right)\right)^{\gamma_{2}}\right)^{k}
$$

and

$$
\mathbb{E}^{x_{0}}\left(L_{\bar{\theta}_{r}}^{\pi \rho \mid D\left(0, r_{1}\right)}\right)=\rho\left(D\left(0, r_{1}\right)\right) \log \left(r / r_{2}\right) \pm c r_{1}^{\gamma_{1}}\left(\log \left(1 / r_{1}\right)\right)^{\gamma_{2}}
$$

Proof of Lemma 2.3. We recall Kac's moment formula 7], which can be derived easily from (2.16):

$$
\mathbb{E}^{x_{0}}\left(L_{\bar{\theta}_{r}}^{\pi \rho \mid D\left(0, r_{1}\right)}\right)^{k}=k ! \pi^{k} \int_{D\left(0, r_{1}\right)^{k}} \prod_{j=1}^{k} g_{r}\left(y_{j-1}, y_{j}\right) d \rho\left(y_{j}\right)
$$

Thus, to prove (2.19) we must show that

$$
\pi \int_{D\left(0, r_{1}\right)} g_{r}\left(x_{0}, y\right) d \rho(y)=\rho\left(D\left(0, r_{1}\right)\right) \log \left(r / r_{2}\right) \pm c r_{1}^{\gamma_{1}}\left(\log \left(1 / r_{1}\right)\right)^{\gamma_{2}}
$$

for all $x_{0}$ with $\left|x_{0}\right|=r_{2}$, and (2.18) will follow from (2.20) using

$$
\begin{aligned}
& \sup _{x \in D\left(0, r_{1}\right)} \pi \int_{D\left(0, r_{1}\right)} g_{r}(x, y) d \rho(y) \\
& \quad \leq \rho\left(D\left(0, r_{1}\right)\right) \log \left(r / r_{1}\right)+c r_{1}^{\gamma_{1}}\left(\log \left(1 / r_{1}\right)\right)^{\gamma_{2}}
\end{aligned}
$$

inductively for the $d y_{k}, d y_{k-1}, \ldots, d y_{2}$ integrals, and (2.21) for the $d y_{1}$ integral.

By (2.3) we can bound the left-hand side in the last display by

$$
\sup _{x \in D\left(0, r_{1}\right)} \int_{D\left(0, r_{1}\right)}\left(\log \left(r / r_{1}\right)+\log \left(\frac{r_{1}}{|y-x|}\right)+\pi c_{o}\right) d \rho(y) .
$$

Now $D\left(0, r_{1}\right) \subseteq D\left(x, e r_{1}\right)$ for any $x \in D\left(0, r_{1}\right)$, and breaking $D\left(x, e r_{1}\right)$ up into annuli $D\left(x, e r_{1}\right)=\bigcup_{j=-1}^{\infty}\left\{D\left(x, e^{-j} r_{1}\right) \backslash D\left(x, e^{-j-1} r_{1}\right)\right\}$ allows us to bound

$$
\begin{aligned}
& \int_{D\left(0, r_{1}\right)}\left|\log \left(\frac{r_{1}}{|x-y|}\right)\right| d \rho(y) \\
& \leq \sum_{j=-1}^{\infty} \int_{D\left(x, e^{-j} r_{1}\right) \backslash D\left(x, e^{-j-1} r_{1}\right)}\left|\log \left(\frac{r_{1}}{|x-y|}\right)\right| d \rho(y) \\
& \leq \sum_{j=-1}^{\infty}|j+2| \rho\left(D\left(x, e^{-j} r_{1}\right)\right) \leq c_{1} \sum_{j=-1}^{\infty}|j+2| e^{-\gamma_{1} j} r_{1}^{\gamma_{1}}\left(\log 1 / r_{1}+|j|\right)^{\gamma_{2}}
\end{aligned}
$$

where we have used the upper bound (2.17) for the last inequality. This immediately gives (2.22).

Turning to (2.21), by (2.3) we can write the left-hand side as

$$
\begin{gathered}
\int_{D\left(0, r_{1}\right)}\left(\log \left(r / r_{2}\right)+\log \left(\frac{r_{2}}{\left|x_{0}-y\right|}\right) \pm \pi c_{o}\right) d \rho(y) \\
=\left(\log \left(r / r_{2}\right) \pm \pi c_{o}\right) \rho\left(D\left(0, r_{1}\right)\right)+\int_{D\left(0, r_{1}\right)} \log \left(\frac{r_{2}}{\left|x_{0}-y\right|}\right) d \rho(y) .
\end{gathered}
$$


When $r_{1} / r_{2} \leq 1 / 2$, we have that $\left|\log \frac{r_{2}}{\left|x_{0}-y\right|}\right|$ is bounded on $D\left(0, r_{1}\right)$ so that (2.21) follows in that case. When $1 / 2 \leq r_{1} / r_{2} \leq 1$, we can use

$$
\int_{D\left(0, r_{1}\right)}\left|\log \left(\frac{r_{2}}{\left|x_{0}-y\right|}\right)\right| d \rho(y) \leq \int_{D\left(0, r_{2}\right)}\left|\log \left(\frac{r_{2}}{\left|x_{0}-y\right|}\right)\right| d \rho(y),
$$

and (2.21) follows by the argument applied in (2.23).

\section{LOWER BOUNDS}

Fixing $a<1, c>0$ and $\delta>0$, let

$$
\Gamma_{c}=\Gamma_{c}\left(\omega, \omega^{\prime}\right):=\left\{x \in D(0,1): \lim _{\varepsilon \rightarrow 0} \frac{\mathcal{I}_{\bar{\theta}_{c}, \bar{\theta}_{c}^{\prime}}(D(x, \varepsilon))}{\varepsilon^{2}\left(\log \frac{1}{\varepsilon}\right)^{4}}=a^{2}\right\},
$$

and $\mathcal{E}_{c}:=\left\{\omega, \omega^{\prime}: \operatorname{dim}\left(\Gamma_{c}\left(\omega, \omega^{\prime}\right)\right) \geq 2-2 a-\delta\right\}$.

In view of the results of Section 2, we will obtain Theorem 1.4 once we show that $\mathbf{P} \times \mathbf{P}^{\prime}\left(\mathcal{E}_{1}\right)=1$ for any $a<1$ and $\delta>0$. Moreover, then the inequality

$$
\liminf _{\epsilon \rightarrow 0} \sup _{|x|<1} \frac{\mathcal{I}_{\bar{\theta}, \bar{\theta}^{\prime}}(D(x, \varepsilon))}{\varepsilon^{2}\left(\log \frac{1}{\varepsilon}\right)^{4}} \geq \sup _{|x|<1} \liminf _{\epsilon \rightarrow 0} \frac{\mathcal{I}_{\bar{\theta}, \bar{\theta}^{\prime}}(D(x, \varepsilon))}{\varepsilon^{2}\left(\log \frac{1}{\varepsilon}\right)^{4}}
$$

implies that for any $\eta>0$,

$$
\liminf _{\varepsilon \rightarrow 0} \sup _{|x|<1} \frac{\mathcal{I}_{\bar{\theta}, \bar{\theta}^{\prime}}(D(x, \varepsilon))}{\varepsilon^{2}\left(\log \frac{1}{\varepsilon}\right)^{4}} \geq 1-\eta, \quad \text { a.s. }
$$

In view of (2.10), these lower bounds establish Theorem 1.3 .

The bulk of this section and the next will be dedicated to showing that $\mathbf{P} \times$ $\mathbf{P}^{\prime}\left(\mathcal{E}_{1}\right)>0$. Assuming this for the moment, let us show that this implies $\mathbf{P} \times$ $\mathbf{P}^{\prime}\left(\mathcal{E}_{1}\right)=1$. With $W_{t}^{c}:=c^{-1} W_{c^{2} t}$ we have that

$$
c^{2} \bar{\theta}\left(\omega^{c}\right)=\inf \left\{c^{2} t:\left|c^{-1} W_{c^{2} t}\right|=1\right\}=\bar{\theta}_{c}(\omega),
$$

and similarly for $W^{\prime}$ and hence

$$
\begin{aligned}
& \mathcal{I}_{\bar{\theta}, \bar{\theta}^{\prime}}^{W^{c}, W^{\prime c}}(D(x, \varepsilon)) \\
& =\lim _{\gamma \rightarrow 0} \pi \int_{0}^{\bar{\theta}\left(\omega^{c}\right)} \int_{0}^{\bar{\theta}\left(\omega^{\prime c}\right)} 1_{\left\{\left|W_{s}^{c}-x\right| \leq \epsilon\right\}} f_{\gamma}\left(W_{s}^{c}-W_{t}^{\prime c}\right) d s d t \\
& =\lim _{\gamma \rightarrow 0} \pi \int_{0}^{\bar{\theta}\left(\omega^{c}\right)} \int_{0}^{\bar{\theta}\left(\omega^{\prime c}\right)} 1_{\left\{\left|W_{c^{2} s}-c x\right| \leq c \epsilon\right\}} f_{\gamma}\left(c^{-1} W_{c^{2} s}-c^{-1} W_{c^{2} t}^{\prime}\right) d s d t \\
& =\lim _{\gamma \rightarrow 0} \frac{\pi}{c^{2}} \int_{0}^{c^{2} \bar{\theta}\left(\omega^{c}\right)} \int_{0}^{c^{2} \bar{\theta}\left(\omega^{\prime c}\right)} 1_{\left\{\left|W_{s}-c x\right| \leq c \epsilon\right\}} f_{c \gamma}\left(W_{s}-W_{t}^{\prime}\right) d s d t \\
& =\frac{1}{c^{2}} \mathcal{I}_{\bar{\theta}_{c}, \bar{\theta}_{c}^{\prime}}(D(c x, c \varepsilon)) .
\end{aligned}
$$

Consequently, $\Gamma_{c}\left(\omega, \omega^{\prime}\right)=c \Gamma_{1}\left(\omega^{c}, \omega^{\prime c}\right)$; so Brownian scaling implies that $p=\mathbf{P} \times$ $\mathbf{P}^{\prime}\left(\mathcal{E}_{c}\right)$ is independent of $c>0$. Let

$$
\mathcal{E}:=\limsup _{n \rightarrow \infty} \mathcal{E}_{n^{-1}}
$$

so that $\mathbf{P} \times \mathbf{P}^{\prime}(\mathcal{E}) \geq p$. Since $\mathcal{E}_{c} \in \mathcal{F}_{\bar{\theta}_{c}} \times \mathcal{F}^{\prime} \bar{\theta}_{c}^{\prime}$ and $\bar{\theta}_{n^{-1}} \downarrow 0$, the Blumenthal $0-1$ law tells us that $\mathbf{P} \times \mathbf{P}^{\prime}(\mathcal{E}) \in\{0,1\}$. Thus, $p>0$ yields $\mathbf{P} \times \mathbf{P}^{\prime}(\mathcal{E})=1$. Since $x \mapsto \mathcal{I}_{\bar{\theta}_{b}, \bar{\theta}_{b}^{\prime}}(D(x, \varepsilon))$ are Borel measurable, it follows that $\Gamma_{b}$ are Borel sets (hence 
analytic), and exactly as in [5, (3.1)] we find that the events $\mathcal{E}_{c}$ are essentially increasing in $c$, i.e.,

$$
\forall 0<b<c \quad \mathbf{P} \times \mathbf{P}^{\prime}\left(\mathcal{E}_{b} \backslash \mathcal{E}_{c}\right)=0 .
$$

Thus, $\mathbf{P} \times \mathbf{P}^{\prime}\left(\mathcal{E} \backslash \mathcal{E}_{1}\right) \leq \mathbf{P} \times \mathbf{P}^{\prime}\left(\bigcup_{n}\left\{\mathcal{E}_{n^{-1}} \backslash \mathcal{E}_{1}\right\}\right)=0$, so that also $\mathbf{P} \times \mathbf{P}^{\prime}\left(\mathcal{E}_{1}\right)=1$.

It thus remains to show that $\mathbf{P} \times \mathbf{P}^{\prime}\left(\mathcal{E}_{1}\right)>0$. We start by constructing a subset of $\Gamma_{1}$, the Hausdorff dimension of which is easier to bound below. To this end we recall some notation from [5]. Fix $a<2, \epsilon_{1}=1 / 8$ and the squares $S=S_{1}=$ $\left[\epsilon_{1}, 2 \epsilon_{1}\right]^{2} \subset D(0,1)$. Note that for all $x \in S$ and $y \in S \cup\{0\}$, both $0 \notin D\left(x, \epsilon_{1}\right)$ and $0 \in D(x, 1 / 2) \subset D(y, 1) \subset D(x, 2)$. Let $\epsilon_{k}=\epsilon_{1}(k !)^{-3}=\epsilon_{1} \prod_{l=2}^{k} l^{-3}$. For $x \in S, k \geq 2$ and $\rho>\epsilon_{1}$, let $N_{k}^{x}(\rho)$ denote the number of excursions of $W$ from $\partial D\left(x, \epsilon_{k-1}\right)$ to $\partial D\left(x, \epsilon_{k}\right)$ prior to hitting $\partial D(x, \rho)$. Set $n_{k}=3 a k^{2} \log k$. We will say that a point $x \in S$ is $n$-perfect if

$$
n_{k}-k \leq N_{k}^{x}(1 / 2) \leq N_{k}^{x}(2) \leq n_{k}+k, \quad \forall k=2, \ldots, n .
$$

For $n \geq 2$ we partition $S$ into $M_{n}=\epsilon_{1}^{2} /\left(2 \epsilon_{n}\right)^{2}=(1 / 4) \prod_{l=1}^{n} l^{6}$ non-overlapping squares of edge length $2 \epsilon_{n}=2 \epsilon_{1} /(n !)^{3}$, which we denote by $S(n, i) ; i=1, \ldots, M_{n}$ with $x_{n, i}$ denoting the center of each $S(n, i)$. Let $Y(n, i) ; i=1, \ldots, M_{n}$ be the sequence of random variables defined by

$$
Y(n, i)=1 \text { if } x_{n, i} \text { is } n \text {-perfect }
$$

and $Y(n, i)=0$ otherwise. Set $q_{n, i}=\mathbf{P}(Y(n, i)=1)=\mathbb{E}(Y(n, i))$. Define

$$
A_{n}=\bigcup_{i: Y(n, i)=1} S(n, i)
$$

and

$$
F=F(\omega)=\bigcap_{m} \overline{\bigcup_{n \geq m} A_{n}}:=\bigcap_{m} F_{m} .
$$

Note that each $x \in F$ is the limit of a sequence $\left\{x_{n}\right\}$ such that $x_{n}$ is $n$-perfect. Finally, we set

$$
C_{a}:=\left\{x \in S: \lim _{\epsilon \rightarrow 0} \frac{\mu_{\bar{\theta}^{\prime}}^{w^{\prime}}(D(x, \varepsilon))}{\varepsilon^{2}(\log \epsilon)^{2}}=a\right\} .
$$

The next lemma (to be proved in Section 4), shows that $F \cap C_{a} \subset \Gamma_{1}$.

Lemma 3.1. A.s. for all $x \in F \cap C_{a}$,

$$
\lim _{\varepsilon \rightarrow 0} \frac{\mathcal{I}_{\bar{\theta}, \bar{\theta}^{\prime}}(D(x, \varepsilon))}{\varepsilon^{2}\left(\log \frac{1}{\varepsilon}\right)^{4}}=a^{2} .
$$

To complete the proof that $\mathbf{P} \times \mathbf{P}^{\prime}\left(\mathcal{E}_{1}\right)>0$, it thus suffices to show that

$$
\mathbf{P} \times \mathbf{P}^{\prime}\left(\operatorname{dim}\left(F \cap C_{a}\right) \geq 2-2 a-2 \delta\right)>0,
$$

for any $a<1$ and $\delta>0$.

It was proved in [5, Section 3] that

$$
\mathbf{P}^{\prime}\left(\operatorname{dim}\left(C_{a}\right) \geq 2-a-\delta\right)>0,
$$

for any $a<2$ and $\delta>0$. The following general lemma will thus imply (3.5). 
Lemma 3.2. Let $B \subseteq S$ be a closed $\mathcal{F}^{\prime}$-measurable set with

$$
\mathbf{P}^{\prime}(\operatorname{dim}(B) \geq b)>0
$$

Then, for any $a<b$ and $\delta>0$,

$$
\mathbf{P} \times \mathbf{P}^{\prime}(\operatorname{dim}(F \cap B) \geq b-a-\delta)>0 .
$$

Proof. Fixing $a<b$ and $\delta>0$ such that $h:=b-a-\delta>0$, we establish (3.7) by finding a set $\mathcal{C}$ of positive $\mathbf{P} \times \mathbf{P}^{\prime}$ probability, such that for any $\left(\omega, \omega^{\prime}\right) \in \mathcal{C}$, we can find a nonzero random measure $\rho_{\omega, \omega^{\prime}}$ supported on $F(\omega) \cap B\left(\omega^{\prime}\right)$ with finite $h$-energy, where the $h$-energy of a measure $\nu$ is defined as

$$
\mathcal{G}_{h}(\nu)=\iint|x-y|^{-h} d \nu(x) d \nu(y)
$$

(see, e.g., [16, Theorem 8.7]).

By (3.6) we can find a set $\mathcal{C}^{\prime}$ with $\mathbf{P}^{\prime}\left(\mathcal{C}^{\prime}\right)>0$ and a finite constant $c$ such that for any $\omega^{\prime} \in \mathcal{C}^{\prime}$, we have a probability measure $\mu_{\omega^{\prime}}$ supported on $B$ with

$$
\mathcal{G}_{b-\delta / 2}\left(\mu_{\omega^{\prime}}\right)<c .
$$

In the sequel, we restrict attention to $\omega^{\prime} \in \mathcal{C}^{\prime}$ without mentioning it explicitly. The measure $\rho=\rho_{\omega, \omega^{\prime}}$ will be constructed as a weak limit of measures $\nu_{n}$, where $\nu_{n}=\nu_{n, \omega, \omega^{\prime}}$ for $n \geq 2$ is the random measure supported on $A_{n} \subseteq F_{n}$ whose density with respect to $\mu_{\omega^{\prime}}$ is

$$
f_{n}(x)=\sum_{i=1}^{M_{n}} q_{n, i}^{-1} 1_{\{Y(n, i)=1\}} 1_{\{x \in S(n, i)\}} .
$$

Note that

$$
\mathbb{E}\left(\nu_{n}(S)\right)=\sum_{i=1}^{M_{n}} q_{n, i}^{-1} \mathbf{P}(Y(n, i)=1) \mu_{\omega^{\prime}}(S(n, i))=\mu_{\omega^{\prime}}\left(\left[\epsilon_{1}, 2 \epsilon_{1}\right]^{2}\right)=1 .
$$

Next we recall Lemma 3.2 of [5], combining it with the comments just below it. In the sequel, we let $C_{m}$ denote generic finite constants that are independent of $n$.

Lemma 3.3. Let $l(i, j)=\min \left\{m: D\left(x_{n, i}, \epsilon_{m}\right) \cap D\left(x_{n, j}, \epsilon_{m}\right)=\emptyset\right\} \leq n$, with $l(i, i):=n$. Then, there exists $\delta_{n} \rightarrow 0$ such that for all $n \geq 2, i$,

$$
q_{n, i} \geq Q_{n}:=\inf _{x \in S} \mathbf{P}(x \text { is } n \text {-perfect }) \geq \epsilon_{n}^{a+\delta_{n}},
$$

whereas for all $n$,

$$
\mathbb{E}(Y(n, i) Y(n, j)) \leq C_{0} Q_{n}^{2} \epsilon_{l(i, j)}^{-a-\delta_{l(i, j)}} .
$$

Furthermore, $Q_{n} \geq c q_{n, i}$ for some $c>0$ and all $n \geq 2$ and $i$. 
It follows from Lemma 3.3 that for all $\omega^{\prime} \in \mathcal{C}^{\prime}$,

$$
\begin{aligned}
& \mathbb{E}\left(\left(\nu_{n}(S)\right)^{2}\right) \\
& \quad=\sum_{i, j=1}^{M_{n}} q_{n, i}^{-1} q_{n, j}^{-1} \mathbb{E}(Y(n, i) Y(n, j)) \mu_{\omega^{\prime}}(S(n, i)) \mu_{\omega^{\prime}}(S(n, j)) \\
& \quad \leq C_{1} \sum_{i, j=1}^{M_{n}} \epsilon_{l(i, j)}^{-a-\delta_{l(i, j)}} \mu_{\omega^{\prime}}(S(n, i)) \mu_{\omega^{\prime}}(S(n, j)) \\
& \quad \leq C_{2} \sum_{i, j=1}^{M_{n}} \int_{x \in S(n, i)} \int_{y \in S(n, j)} \epsilon_{l(i, j)}^{-b+\delta-\delta_{l(i, j)}} \mu_{\omega^{\prime}}(d x) \mu_{\omega^{\prime}}(d y) \\
& \leq C_{3} \iint|x-y|^{-b+\delta / 2} \mu_{\omega^{\prime}}(d x) \mu_{\omega^{\prime}}(d y)=C_{3} \mathcal{G}_{b-\delta / 2}\left(\mu_{\omega^{\prime}}\right) \\
& \leq C_{3} c<\infty
\end{aligned}
$$

is a bounded sequence (recall that $\delta_{l} \rightarrow 0$ ). Applying the Paley-Zygmund inequality (see 9, page 8]), (3.8) and (3.9) together guarantee that for some $r>0, v>0$ and all $\omega^{\prime} \in \mathcal{C}^{\prime}$,

$$
\mathbf{P}\left(r^{-1} \geq \nu_{n}(S) \geq r\right) \geq 2 v>0, \quad \forall n .
$$

Similarly, for $h=b-a-\delta \in(0,2)$, and $\omega^{\prime} \in \mathcal{C}^{\prime}$,

$$
\begin{aligned}
& \mathbb{E}\left(\mathcal{G}_{h}\left(\nu_{n}\right)\right) \\
& \quad \leq C_{4} \sum_{i, j=1}^{M_{n}} \frac{\mathbb{E}(Y(n, i) Y(n, j))}{q_{n, i} q_{n, j}} \int_{S(n, i)} \int_{S(n, j)}|x-y|^{-h} \mu_{\omega^{\prime}}(d x) \mu_{\omega^{\prime}}(d y) \\
& \quad \leq C_{5} \sum_{i, j=1}^{M_{n}} \epsilon_{l(i, j)}^{-a-\delta_{l(i, j)}} \int_{S(n, i)} \int_{S(n, j)}|x-y|^{-h} \mu_{\omega^{\prime}}(d x) \mu_{\omega^{\prime}}(d y) \\
& \leq C_{6} \mathcal{G}_{b-\delta / 2}\left(\mu_{\omega^{\prime}}\right) \leq C_{6} c<\infty
\end{aligned}
$$

is a bounded sequence. Thus we can find $d<\infty$ such that for all $\omega^{\prime} \in \mathcal{C}^{\prime}$,

$$
\mathbf{P}\left(\mathcal{G}_{h}\left(\nu_{n}\right) \leq d\right) \geq 1-v>0, \quad \forall n .
$$

Combined with (3.10) this shows that for all $\omega^{\prime} \in \mathcal{C}^{\prime}$,

$$
\mathbf{P}\left(r^{-1} \geq \nu_{n}(S) \geq r, \mathcal{G}_{h}\left(\nu_{n}\right) \leq d\right) \geq v>0, \quad \forall n .
$$

Let $\mathcal{C}_{n}=\left\{\left(\omega, \omega^{\prime}\right): r^{-1} \geq \nu_{n}(S) \geq r, \mathcal{G}_{h}\left(\nu_{n}\right) \leq d, \omega^{\prime} \in \mathcal{C}^{\prime}\right\}$ and $\operatorname{set} \mathcal{C}=\lim \sup _{n} \mathcal{C}_{n}$. Then (3.11) implies that

$$
\mathbf{P} \times \mathbf{P}^{\prime}(\mathcal{C}) \geq v>0 .
$$

Fixing $\left(\omega, \omega^{\prime}\right) \in \mathcal{C}$ there exists a subsequence $n_{k} \rightarrow \infty$ such that $\left(\omega, \omega^{\prime}\right) \in \mathcal{C}_{n_{k}}$ for all $k$. Due to the lower semi-continuity of $\mathcal{G}_{h}(\cdot)$, the set of nonnegative measures $\nu$ on $S$ such that $\nu(S) \in\left[r, r^{-1}\right]$ and $\mathcal{G}_{h}(\nu) \leq d$ is compact with respect to weak convergence. Thus, for $\left(\omega, \omega^{\prime}\right) \in \mathcal{C}$, the sequence $\nu_{n_{k}}=\nu_{n_{k}, \omega, \omega^{\prime}}$ has at least one weak limit $\rho_{\omega, \omega^{\prime}}$ which is a finite measure supported on $F(\omega) \cap B\left(\omega^{\prime}\right)$, having positive mass and finite $h$-energy. This completes the proof of Lemma 3.2. 
Let $\left\{X_{t}\right\}$ denote the symmetric stable process of index $\beta$, with law denoted $\mathbf{P}^{\prime}$. Fix $T<\infty$ and for $a<\beta / 2$, define

$$
C_{a}^{X}=\left\{x \in S: \limsup _{\epsilon \rightarrow 0} \frac{\mu_{T}^{X}(D(x, \epsilon))}{\Lambda \epsilon^{\beta}|\log \epsilon|}=a\right\} .
$$

As in the case of two Brownian motions, the lower bound in Theorem 1.6 follows from two results. The first result is the next lemma, which is proved in Section 4 ,

Lemma 3.4. A.s. for all $x \in F \cap C_{a}^{X}$,

$$
\limsup _{\varepsilon \rightarrow 0} \frac{\mathcal{I}_{\bar{\theta}, T}^{W, X}(D(x, \varepsilon))}{\varepsilon^{\beta}\left(\log \frac{1}{\varepsilon}\right)^{3}}=a^{2} .
$$

The second result says that for any $\delta>0$,

$$
\mathbf{P} \times \mathbf{P}^{\prime}\left(\operatorname{dim}\left(F \cap C_{a}^{X}\right) \geq \beta-2 a-\delta\right)>0 .
$$

Indeed, recall from [4, (1.5)] that $\mathbf{P}^{\prime}\left(\operatorname{dim}\left(C_{a}^{X}\right)=\beta-a\right)=1$ (by shift invariance and stable scaling, [4, (1.5)] is valid even when restricting to $x \in S$ ). We can extract a closed subset of $C_{a}^{X}$ which still has dimension $\beta-a$, so that (3.13) follows from Lemma 3.2

As in the case of two Brownian motions, we conclude from (3.12) and (3.13) that $\mathbf{P} \times \mathbf{P}^{\prime}\left(\mathcal{E}_{1}\right)>0$, where $\mathcal{E}_{c}:=\left\{\omega, \omega^{\prime}: \operatorname{dim}\left(\Gamma_{c}\left(\omega, \omega^{\prime}\right)\right) \geq \beta-2 a-\delta\right\}$ and

$$
\Gamma_{c}\left(\omega, \omega^{\prime}\right):=\left\{x \in D(0, c): \limsup _{\varepsilon \rightarrow 0} \frac{\mathcal{I}_{\bar{\theta}_{c}, c^{\beta} T}^{W, X}(D(x, \varepsilon))}{\varepsilon^{\beta}\left(\log \frac{1}{\varepsilon}\right)^{3}}=a^{2}\right\} .
$$

With $W_{t}^{c}:=c^{-1} W_{c^{2} t}$ and $X_{t}^{c}:=c^{-1} X_{c^{\beta} t}$, it follows by Brownian and stable scaling that

$$
\mathcal{I}_{\bar{\theta}, T}^{W^{c}, X^{c}}(D(x, \varepsilon))=c^{-\beta} \mathcal{I}_{\bar{\theta}_{c}, c^{\beta} T}^{W, X}(D(c x, c \varepsilon))
$$

(see (3.1) for a similar derivation). Consequently, $\Gamma_{c}\left(\omega, \omega^{\prime}\right)=c \Gamma_{1}\left(\omega^{c}, \omega^{\prime c}\right)$, with Brownian and stable scaling together implying that $p=\mathbf{P} \times \mathbf{P}^{\prime}\left(\mathcal{E}_{c}\right)>0$ is independent of $c>0$. Since $\mathcal{E}_{c} \in \mathcal{F}_{\bar{\theta}_{c}} \times \sigma\left(X_{t}: 0 \leq t \leq c^{\beta} T\right)$, and $\bar{\theta}_{n^{-1}} \downarrow 0$, the Blumenthal $0-1$ law tells us that $\mathbf{P} \times \mathbf{P}^{\prime}\left(\lim \sup _{n \rightarrow \infty} \mathcal{E}_{n^{-1}}\right)=1$, resulting with $\mathbf{P} \times \mathbf{P}^{\prime}\left(\mathcal{E}_{1}\right)=1$ (see (3.2) for more details). This concludes the proof of the lower bound for any $T<\infty$. Next note that the random set $\Gamma_{1}$ for $T=\infty$ is the same as $\Gamma_{1}$ for $T<\infty$ whenever $\inf _{s \geq T}\left|X_{s}\right|>1$. Consequently, in case $T=\infty$, we see that

$$
\mathbf{P} \times \mathbf{P}^{\prime}\left(\mathcal{E}_{1}\right) \geq \lim _{T \rightarrow \infty} \mathbf{P}^{\prime}\left(\inf _{s \geq T}\left|X_{s}\right|>1\right)=1
$$

(by stable scaling and the transience of $X_{t}$ ).

Remark. The alert reader might ask whether it is possible to get a statement similar to the one in Lemma 3.2 but rather holding with probability one. Recall that

$$
\operatorname{Thick}_{a}^{W}=\left\{x \in D(0,1): \lim _{\epsilon \rightarrow 0} \frac{\mu_{\bar{\theta}}^{W}(D(x, \epsilon))}{\epsilon^{2}(\log \epsilon)^{2}}=a\right\} .
$$

A variation of our proof yields the following statement:

Let $B \subset D(0,1)$ be a (possibly random) closed set, independent of Thick ${ }_{a}^{W}$. Furthermore, assume there exist random probability measures $\left\{\mu_{n, \omega^{\prime}}(\cdot)\right\}$ such that 
$\mathbf{P}^{\prime}\left(\mathcal{G}_{b}\left(\mu_{1, \omega^{\prime}}\right)<\infty\right)=1, \mu_{n, \omega^{\prime}}(\cdot)$ possesses the same law as $\mu_{1, \omega^{\prime}}\left(\cdot / 2^{n-1}\right)$, and for any $n \geq 1$,

$$
\mathbf{P}^{\prime}\left(\mu_{n, \omega^{\prime}}\left(B \cap D\left(0,2^{-(n-1)}\right)\right)=1\right)=1 .
$$

Finally, assume that the sequence of measures $\mu_{n, \omega^{\prime}}$, viewed as measure-valued random variables, possesses a trivial tail. Then,

$$
\mathbf{P} \times \mathbf{P}^{\prime}\left(\operatorname{dim}\left(\text { Thick }_{a}^{W} \cap B\right) \geq b-a\right)=1 .
$$

\section{FROM EXCURSIONS TO INTERSECTION LOCAL TIMES}

4.1. Intersection of two Brownian motions. Recall the sets $F, C_{a}$, introduced in (3.3) and (3.4) and $h(\varepsilon)=\varepsilon^{2}(\log \varepsilon)^{4}$. Lemma 3.1 will follow from the next two lemmas.

Lemma 4.1. For every $\delta>0$, if $x \in F \cap C_{a}$, then

$$
a^{2}(1-\delta)^{5} \leq \liminf _{\epsilon \rightarrow 0} \mathcal{I}_{\bar{\theta}, \bar{\theta}^{\prime}}(D(x, \epsilon)) / h(\epsilon) .
$$

Lemma 4.2. For every $\delta>0$, if $x \in F \cap C_{a}$, then

$$
\limsup _{\epsilon \rightarrow 0} \mathcal{I}_{\bar{\theta}, \bar{\theta}^{\prime}}(D(x, \epsilon)) / h(\epsilon) \leq a^{2}(1+\delta)^{5} .
$$

Proof of Lemma 4.1. Let $\delta_{k}=\epsilon_{k} / k^{6}$ and let $\mathcal{D}_{k}$ be a $\delta_{k}$-net of points in $S$. Let

$$
\epsilon_{k}^{\prime}=\epsilon_{k} e^{1 / k^{6}}, \quad \epsilon_{k-1}^{\prime \prime}=\epsilon_{k-1} e^{-1 / k^{6}}
$$

so that

$$
\epsilon_{k}^{\prime} \geq \epsilon_{k}+\delta_{k}, \quad \epsilon_{k-1}^{\prime \prime} \leq \epsilon_{k-1}-\delta_{k}
$$

We will say that a point $x^{\prime} \in \mathcal{D}_{k}$ is lower $k$-successful if there are at least $n_{k}-k$ excursions of $W$ from $\partial D\left(x^{\prime}, \epsilon_{k-1}^{\prime \prime}\right)$ to $\partial D\left(x^{\prime}, \epsilon_{k}^{\prime}\right)$ prior to $\bar{\theta}$. Let

$$
\epsilon_{k, j}=\epsilon_{k} e^{-j / k}, j=0,1, \ldots, 3 k \log (k+1),
$$

and let $\epsilon_{k, j}^{\prime}=\epsilon_{k, j} e^{-2 / k^{3}}=\epsilon_{k}^{\prime} e^{-j / k} e^{-2 / k^{3}-1 / k^{6}}$. We say that $x^{\prime} \in \mathcal{D}_{k}$ is lower $k$, $\delta$-successful if it is lower $k$-successful and, in addition,

$$
a(1-\delta) \epsilon_{k, j}^{\prime 2}\left|\log \epsilon_{k, j}^{\prime}\right|^{2} \leq \mu_{\bar{\theta}^{\prime}}^{W^{\prime}}\left(D\left(x^{\prime}, \epsilon_{k, j}^{\prime}\right)\right), \forall j=0, \ldots, 3 k \log (k+1) .
$$

We now derive Lemma 4.1 from the following lemma.

Lemma 4.3. There exists a $k_{0}=k_{0}\left(\delta, \omega, \omega^{\prime}\right)$ such that for all $k \geq k_{0}$ and $x^{\prime} \in \mathcal{D}_{k}$, if $x^{\prime}$ is lower $k, \delta$-successful, then

$$
a^{2}(1-\delta)^{4} h\left(\epsilon_{k, j}^{\prime}\right) \leq \mathcal{I}_{\bar{\theta}, \bar{\theta}^{\prime}}\left(D\left(x^{\prime}, \epsilon_{k, j}^{\prime}\right)\right), \quad \forall j=0,1, \ldots, 3 k \log (k+1) .
$$

To begin our derivation of Lemma 4.1 note that if $x \in F$, then there exists a sequence of $n$-perfect points $x_{n} \rightarrow x$. Since an $n$-perfect point is $k$-perfect for all $k \leq n$, it follows that one may find a point $\tilde{x}_{k}$ that is $k$-perfect and satisfies $\left|x-\tilde{x}_{k}\right|<\epsilon_{k} / k^{6}$. Let $x_{k} \in \mathcal{D}_{k}$ with $\left|x_{k}-\tilde{x}_{k}\right| \leq \epsilon_{k} / k^{6}$. Using (4.3) and the fact that $\tilde{x}_{k}$ is $k$-perfect we can see that $x_{k} \in \mathcal{D}_{k}$ is lower $k$-successful. (cf. [5, Section 6 , figure 1]).

Since $\left|x-x_{k}\right| \leq 2 \epsilon_{k} / k^{6}$, we have

$$
\mu_{\bar{\theta}^{\prime}}^{W^{\prime}}\left(D\left(x_{k}, \epsilon_{k, j}^{\prime}\right)\right) \geq \mu_{\bar{\theta}^{\prime}}^{W^{\prime}}\left(D\left(x, \epsilon_{k, j}^{\prime}-2 \epsilon_{k} / k^{6}\right)\right)
$$

for all $j, k$, so that if $x \in C_{a}$, it is easy to see that there exists a $k_{2}=k_{2}(x, \omega, \delta)$ such that for $k>k_{2}, x_{k}$ is in fact lower $k, \delta$-successful. 
Applying Lemma 4.3 with $x^{\prime}=x_{k}$ and using $\epsilon_{k, j}^{\prime}+2 \epsilon_{k} / k^{6} \leq \epsilon_{k, j}$ so that

$$
\mathcal{I}_{\bar{\theta}, \bar{\theta}^{\prime}}\left(D\left(x_{k}, \epsilon_{k, j}^{\prime}\right)\right) \leq \mathcal{I}_{\bar{\theta}, \bar{\theta}^{\prime}}\left(D\left(x, \epsilon_{k, j}^{\prime}+2 \epsilon_{k} / k^{6}\right)\right) \leq \mathcal{I}_{\bar{\theta}, \bar{\theta}^{\prime}}\left(D\left(x, \epsilon_{k, j}\right)\right)
$$

then shows that for all $k$ sufficiently large,

$$
a^{2}(1-\delta)^{4} h\left(\epsilon_{k, j}\right) \leq \mathcal{I}_{\bar{\theta}, \bar{\theta}^{\prime}}\left(D\left(x, \epsilon_{k, j}\right)\right), \quad \forall j=0,1, \ldots, 3 k \log (k+1) .
$$

Now for any $\epsilon_{k+1} \leq \epsilon \leq \epsilon_{k}$, let $j$ be such that $\epsilon_{k, j+1} \leq \epsilon \leq \epsilon_{k, j}$. Then

$$
\frac{\mathcal{I}_{\bar{\theta}, \bar{\theta}^{\prime}}(D(x, \epsilon))}{h(\epsilon)} \geq \frac{\mathcal{I}_{\bar{\theta}, \bar{\theta}^{\prime}}\left(D\left(x, \epsilon_{k, j+1}\right)\right)}{h\left(\epsilon_{k, j}\right)} \geq \frac{\mathcal{I}_{\bar{\theta}, \bar{\theta}^{\prime}}\left(D\left(x, \epsilon_{k, j+1}\right)\right)}{h\left(\epsilon_{k, j+1}\right)}(1-4 / k),
$$

and this completes the proof of Lemma 4.1 .

Proof of Lemma 4.3. Suppose that $x^{\prime} \in \mathcal{D}_{k}$ is $k, \delta$-successful. Then there are at least $n_{k}^{\prime}=n_{k}-k$ excursions of $W$ between $\partial D\left(x^{\prime}, \epsilon_{k}^{\prime}\right)$ and $\partial D\left(x^{\prime}, \epsilon_{k-1}^{\prime \prime}\right)$, where $n_{k}^{\prime} \rightarrow \infty$ as $k \rightarrow \infty$. Let $\tau_{l, k, j}$ denote the projected intersection local time measure of $D\left(x^{\prime}, \epsilon_{k, j}^{\prime}\right) \subset D\left(x^{\prime}, \epsilon_{k}^{\prime}\right)$ accumulated while $W$ executes its $l$ th excursion between $\partial D\left(x^{\prime}, \epsilon_{k}^{\prime}\right)$ and $\partial D\left(x^{\prime}, \epsilon_{k-1}^{\prime \prime}\right)$, and $W^{\prime}$ runs from 0 until $\bar{\theta}^{\prime}$. We denote the starting point of the $l$-th such excursion by $x_{l}$.

Let

$$
A\left(x^{\prime}, k, j\right)=\left\{\mathcal{I}_{\bar{\theta}, \bar{\theta}^{\prime}}\left(D\left(x^{\prime}, \epsilon_{k, j}^{\prime}\right)\right) \leq a^{2}(1-\delta)^{4} h\left(\epsilon_{k, j}^{\prime}\right)\right\} .
$$

Using $P^{W}$ to denote probability with respect to $W$, i.e., conditional on $W^{\prime}$, we have

$$
\begin{aligned}
P_{x^{\prime}, k, j} & :=\mathbf{P}^{W}\left(A\left(x^{\prime}, k, j\right), x^{\prime} \text { is lower } k, \delta \text {-successful }\right) \\
& \leq \mathbf{P}_{x^{\prime}, s}^{W}\left(\sum_{l=1}^{n_{k}^{\prime}} \tau_{l, k, j} \leq a^{2}(1-\delta)^{4} h\left(\epsilon_{k, j}^{\prime}\right)\right)
\end{aligned}
$$

where the subscript $x^{\prime}, s$ on $P^{W}$ indicates that $x^{\prime}$ satisfies (4.4). In [5. Theorem 1.2 ] we show that

$$
\lim _{\varepsilon \rightarrow 0} \sup _{x \in \mathbb{R}^{2}} \frac{\mu_{\bar{\theta}_{r}^{\prime}}^{W^{\prime}}(D(x, \varepsilon))}{\varepsilon^{2}\left(\log \frac{1}{\varepsilon}\right)^{2}}=2, \quad \text { a.s. }
$$

Consequently, the measure $\rho(\cdot)=\mu_{\bar{\theta}^{\prime}}^{W^{\prime}}\left(x^{\prime}+\cdot\right)$ satisfies (2.17) for $\gamma_{1}=\gamma_{2}=2$. We now apply Lemma 2.3 with $r_{1}=\epsilon_{k, j}^{\prime}, r_{2}=\epsilon_{k}^{\prime}, r=\epsilon_{k-1}^{\prime \prime}$ and $\rho(\cdot)$ as above. Unfortunately, conditional on $W^{\prime}$, the $\tau_{l, k, j}$ are not i.i.d., and we need to use appropriately the Markov property by conditioning on the starting points of the excursions.

Our condition (4.4) implies that

$$
\rho\left(D\left(0, r_{1}\right)\right)=\mu_{\bar{\theta}^{\prime}}^{W^{\prime}}\left(D\left(x^{\prime}, \epsilon_{k, j}^{\prime}\right)\right) \geq a(1-\delta) r_{1}^{\gamma_{1}}\left(\log \left(1 / r_{1}\right)\right)^{\gamma_{2}},
$$

and so, by (2.19), for all $k \geq k_{1}(a, \delta)$, and denoting by $E_{x^{\prime}, s}^{W, l}$ the expectation over the $l$-th excursion with starting point $x_{l}$, it follows that, uniformly over $l$,

$$
E_{x^{\prime}, s}^{W, l}\left(\tau_{l, k, j}\right)=\mathbb{E}^{x_{l}}\left(L_{\bar{\theta}_{r}}^{\pi \rho \mid D\left(0, r_{1}\right)}\right) \geq a(1-\delta)^{2} \log \left(r / r_{2}\right) r_{1}^{\gamma_{1}}\left(\log \left(1 / r_{1}\right)\right)^{\gamma_{2}} .
$$

Using Stirling's approximation for $\log \epsilon_{k}=\log \epsilon_{1}-3 \log k$ !, it follows that for all $k \geq k_{2}(a, \delta)$ and all $j=0, \ldots, 3 k \log (k+1)$,

$$
n_{k}^{\prime} \log \left(r / r_{2}\right)=n_{k}^{\prime}\left(3 \log k-2 k^{-6}\right) \geq a(1-\delta)\left|\log \left(\epsilon_{k, j}^{\prime}\right)\right|^{2} .
$$

Combining (4.5) and (4.6), we see that

$$
a^{2}(1-\delta)^{3} h\left(\epsilon_{k, j}^{\prime}\right) \leq n_{k}^{\prime} E_{x^{\prime}, s}^{W, l}\left(\tau_{l, k, j}\right) .
$$


This immediately results in

$$
P_{x^{\prime}, k, j} \leq \mathbf{P}_{x^{\prime}, s}^{W}\left(\frac{1}{n_{k}^{\prime}} \sum_{l=1}^{n_{k}^{\prime}} \widehat{\tau}_{l, k, j} \leq 1-\delta\right),
$$

where $\widehat{\tau}_{l, k, j}:=\tau_{l, k, j} / E_{x^{\prime}, s}^{W, l}\left(\tau_{l, k, j}\right)$. Applying Lemma 2.3 as above, we see that for all $k$ large enough,

$$
E_{x^{\prime}, s}^{W, l}\left(\widehat{\tau}_{l, k, j}\right)=1, \quad E_{x^{\prime}, s}^{W, l}\left(\widehat{\tau}_{l, k, j}^{2}\right) \leq 10,
$$

so that, with $\widetilde{\tau}_{l, k, j}:=\widehat{\tau}_{l, k, j}-E_{x^{\prime}, s}^{W, l}\left(\widehat{\tau}_{l, k, j}\right)$, we have

$$
P_{x^{\prime}, k, j} \leq \mathbf{P}_{x^{\prime}, s}^{W}\left(\frac{1}{n_{k}^{\prime}} \sum_{l=1}^{n_{k}^{\prime}} \widetilde{\tau}_{l, k, j} \leq-\delta\right) .
$$

Since $\widetilde{\tau}_{l, k, j} \geq-1$, it follows that for all $0<\theta<1$,

$$
E_{x^{\prime}, s}^{W, l}\left(e^{-\theta \widetilde{\tau}_{l, k, j}}\right) \leq 1+2 \theta^{2} E_{x^{\prime}, s}^{W, l}\left(\widetilde{\tau}_{l, k, j}^{2}\right) \leq 1+20 \theta^{2} \leq e^{20 \theta^{2}} .
$$

Taking $\theta=\delta / 40$, a standard application of Chebyscheff's inequality and the Markov property then shows that for some $\lambda=\lambda(a, \delta)>0, C_{1}<\infty$ and all $x^{\prime} \in \mathcal{D}_{k}, k, j$,

$$
P_{x^{\prime}, k, j} \leq C_{1} e^{-\lambda k^{2} \log k} .
$$

Since $\left|\mathcal{D}_{k}\right| \leq e^{C_{2} k \log k}$ for some $C_{2}<\infty$ and all $k$, it follows that

$$
\sum_{k=1}^{\infty} \sum_{j=0}^{3 k \log (k+1)} \sum_{x^{\prime} \in \mathcal{D}_{k}} P_{x^{\prime}, k, j} \leq 3 C_{1} \sum_{k=1}^{\infty} k^{2} e^{C_{2} k \log k} e^{-\lambda k^{2} \log k}<\infty .
$$

The Borel-Cantelli lemma completes the proof of Lemma 4.3 .

Proof of Lemma 4.2. The situation here is quite similar to the lower bound. Now let

$$
\bar{\epsilon}_{k}^{\prime}=\epsilon_{k} e^{-2 / k^{6}}, \quad \bar{\epsilon}_{k-1}^{\prime \prime}=\epsilon_{k-1} e^{1 / k^{6}},
$$

so that

$$
\bar{\epsilon}_{k}^{\prime} \leq \epsilon_{k}-\delta_{k}, \quad \bar{\epsilon}_{k-1}^{\prime \prime} \geq \epsilon_{k-1}+\delta_{k} .
$$

We now say that $x^{\prime} \in \mathcal{D}_{k}$ is upper $k$-successful if there are at most $n_{k}+k$ excursions of $W$ from $\partial D\left(x^{\prime}, \bar{\epsilon}_{k-1}^{\prime \prime}\right)$ to $\partial D\left(x^{\prime}, \bar{\epsilon}_{k}^{\prime}\right)$ prior to $\bar{\theta}$. We say it is upper $k, \delta$-successful if it is upper $k$-successful and, in addition,

$$
a(1+\delta) \epsilon_{k, j}^{\prime 2}\left|\log \epsilon_{k, j}^{\prime}\right|^{2} \geq \mu_{\bar{\theta}^{\prime}}^{W^{\prime}}\left(D\left(x^{\prime}, \epsilon_{k, j}^{\prime}\right)\right), \quad \forall j=0, \ldots, 3 k \log (k+1) .
$$

As above, we can derive Lemma 4.2 from the following lemma.

Lemma 4.4. There exists a $k_{0}=k_{0}\left(\delta, \omega, \omega^{\prime}\right)$ such that for all $k \geq k_{0}$ and $x^{\prime} \in \mathcal{D}_{k}$, if $x^{\prime}$ is upper $k, \delta$-successful, then

$$
a^{2}(1+\delta)^{4} h\left(\epsilon_{k, j}^{\prime}\right) \geq \mathcal{I}_{\bar{\theta}, \bar{\theta}^{\prime}}\left(D\left(x^{\prime}, \epsilon_{k, j}^{\prime}\right)\right), \quad \forall j=0,1, \ldots, 3 k \log (k+1) .
$$

Since $0 \notin D\left(x, \epsilon_{1}\right)$ for all $x \in S$, with $n_{k}^{\prime \prime}=n_{k}+k$, the proof of Lemma 4.4 in analogy to that of Lemma 4.3 comes down to bounding

$$
Q_{x^{\prime}, k, j}:=\mathbf{P}_{x^{\prime}, u s}^{W}\left(\sum_{l=1}^{n_{k}^{\prime \prime}} \tau_{l, k, j} \geq a^{2}(1+\delta)^{4} h\left(\epsilon_{k, j}^{\prime}\right)\right)
$$


where the subscript $x^{\prime}$, us indicates that $x^{\prime}$ satisfies (4.7). Next we apply Lemma [2.3] for $r_{1}=\epsilon_{k, j}^{\prime}, r_{2}=\bar{\epsilon}_{k}^{\prime}, r=\bar{\epsilon}_{k-1}^{\prime \prime}$ and $\rho(\cdot)=\mu_{\bar{\theta}^{\prime}}^{W^{\prime}}\left(x^{\prime}+\cdot\right)$. Combining (2.19) and condition (4.7), it follows that for all $k \geq k_{3}(a, \delta)$, all $j=0, \ldots, 3 k \log (k+1)$, and all $l$,

$$
E_{x^{\prime}, u s}^{W, l}\left(\tau_{l, k, j}\right) \leq a(1+\delta)^{2} \log \left(\bar{\epsilon}_{k-1}^{\prime \prime} / \bar{\epsilon}_{k}^{\prime}\right)\left(\epsilon_{k, j}^{\prime}\right)^{2}\left|\log \epsilon_{k, j}^{\prime}\right|^{2},
$$

and that

$$
n_{k}^{\prime \prime} \log \left(\bar{\epsilon}_{k-1}^{\prime \prime} / \bar{\epsilon}_{k}^{\prime}\right) \leq a(1+\delta)\left|\log \left(\epsilon_{k, j}^{\prime}\right)\right|^{2}
$$

Consequently,

$$
a^{2}(1+\delta)^{3} h\left(\epsilon_{k, j}^{\prime}\right) \geq n_{k}^{\prime \prime} E_{x^{\prime}, u s}^{W, l}\left(\tau_{l, k, j}\right)
$$

and with $\log \left(\bar{\epsilon}_{k-1}^{\prime \prime} / \bar{\epsilon}_{k}^{\prime}\right) \geq(1 / 3) \log \left(\bar{\epsilon}_{k-1}^{\prime \prime} / \epsilon_{k, j}^{\prime}\right.$ ), it follows that for some $c_{2}>0$ (for example, $c_{2}=a \delta / 3$ will do), and all such $k, j$,

$$
Q_{x^{\prime}, k, j} \leq \mathbf{P}_{x^{\prime}, u s}^{W}\left(\frac{1}{n_{k}^{\prime \prime}} \sum_{l=1}^{n_{k}^{\prime \prime}} \widetilde{\tau}_{l, k, j} \geq c_{2}\right) \leq e^{-c_{2} \lambda n_{k}^{\prime \prime}}\left(E_{x^{\prime}, u s}^{W}\left(e^{\lambda \widetilde{\tau}_{1, k, j}}\right)\right)^{n_{k}^{\prime \prime}}
$$

where now

$$
\widetilde{\tau}_{l, k, j}:=\widehat{\tau}_{l, k, j}-E_{x^{\prime}, u s}^{W, l}\left(\widehat{\tau}_{l, k, j}\right), \quad \widehat{\tau}_{l, k, j}:=\frac{\tau_{l, k, j}}{\log \left(\bar{\epsilon}_{k-1}^{\prime \prime} / \epsilon_{k, j}^{\prime}\right) \epsilon_{k, j}^{\prime 2}\left|\log \epsilon_{k, j}^{\prime}\right|^{2}} .
$$

Applying Lemma 2.3], it follows from (2.17) and (2.18) that for some $C<\infty$, all $\lambda>0$ small and $k$ large enough,

$$
\begin{aligned}
\max _{l} E_{x^{\prime}, u s}^{W, l}\left(e^{\lambda \widetilde{\tau}_{1, k, j}}\right) & =1+\sum_{n=2}^{\infty} \frac{\lambda^{n}}{n !} \max _{l} E_{x^{\prime}, u s}^{W, l}\left(\widetilde{\tau}_{1, k, j}^{n}\right) \\
& \leq 1+\sum_{n=2}^{\infty} \frac{(2 \lambda)^{n}}{n !} \max _{l} E_{x^{\prime}, u s}^{W, l}\left(\widehat{\tau}_{1, k, j}^{n}\right) \leq 1+C \lambda^{2} .
\end{aligned}
$$

The proof of Lemma 4.4 then follows as in the proof of Lemma4.3,

4.2. Intersection with stables. Recall the sets $F, C_{a}^{X}$ introduced in Section 3 In this subsection, we take $T \in(0, \infty)$ and $h(\epsilon)=\epsilon^{\beta}|\log \varepsilon|^{3}$. Lemma 3.4 is implied by the next two lemmas.

Lemma 4.5. For every $\delta>0$, if $x \in F \cap C_{a}^{X}$, then

$$
a^{2}(1-\delta)^{5} \leq \limsup _{\epsilon \rightarrow 0} \mathcal{I}_{\bar{\theta}, T}^{W, X}(D(x, \epsilon)) / h(\epsilon) .
$$

Lemma 4.6. For every $\delta>0$, if $x \in F \cap C_{a}^{X}$, then

$$
\limsup _{\epsilon \rightarrow 0} \mathcal{I}_{\bar{\theta}, T}^{W, X}(D(x, \epsilon)) / h(\epsilon) \leq a^{2}(1+\delta)^{5} .
$$

Proof of Lemma 4.5. Recall the notation $\epsilon_{k}, \epsilon_{k, j}$, etc. of Lemma 4.1 and say now that $x^{\prime} \in \mathcal{D}_{k}$ is lower $k, \delta$-successful if it is lower $k$-successful and

$$
\Lambda a(1-\delta)\left(\epsilon_{k, j}^{\prime}\right)^{\beta}\left|\log \epsilon_{k, j}^{\prime}\right| \leq \mu_{T}^{X}\left(D\left(x^{\prime}, \epsilon_{k, j}^{\prime}\right)\right),
$$

for some $j \in\{0,1, \ldots, 3 k \log (k+1)\}$. In [4, Theorem 1.1] we show that a.s.

$$
\lim _{\epsilon \rightarrow 0} \sup _{|x| \leq 2} \frac{\mu_{T}^{X}(D(x, \epsilon))}{\Lambda \epsilon^{\beta}|\log \epsilon|}=\beta<\infty .
$$


Thus, following the arguments of the proof of Lemma 4.3, but now applying Lemma 2.3 for $\rho(\cdot)=\Lambda^{-1} \mu_{T}^{X}\left(x^{\prime}+\cdot\right)$ with $\gamma_{1}=\beta$ and $\gamma_{2}=1$ (instead of the scale $\gamma_{1}=\gamma_{2}=2$ used in proving Lemma 4.3), we see that there exists a $k_{0}=k_{0}\left(\delta, \omega, \omega^{\prime}\right)$ such that for all $k \geq k_{0}$ and $x^{\prime} \in \mathcal{D}_{k}$, if $x^{\prime}$ is lower $k, \delta$-successful, then

$$
a^{2}(1-\delta)^{4} h\left(\epsilon_{k, j}^{\prime}\right) \leq \mathcal{I}_{\bar{\theta}, T}^{W, X}\left(D\left(x^{\prime}, \epsilon_{k, j}^{\prime}\right)\right)
$$

for some $j \in\{0,1, \ldots, 3 k \log (k+1)\}$. If $x \in F$, then there exists a sequence of points $x_{k} \in \mathcal{D}_{k}$ with $\left|x_{k}-x\right| \leq 2 \epsilon_{k} / k^{6}$ such that $x_{k}$ is lower $k$-successful. If, further, $x \in C_{a}^{X}$, then there exist a subsequence $k_{n} \rightarrow \infty$ and $j_{n} \in\left\{0,1, \ldots, 3 k_{n} \log \left(k_{n}+1\right)\right\}$ such that

$$
\Lambda a(1-\delta)\left(\epsilon_{k_{n}, j_{n}}^{\prime}\right)^{\beta}\left|\log \epsilon_{k_{n}, j_{n}}^{\prime}\right| \leq \mu_{T}^{X}\left(D\left(x_{k_{n}}, \epsilon_{k_{n}, j_{n}}^{\prime}\right)\right) .
$$

Applying (4.10) and using the continuity of $h(\cdot)$, one concludes the proof of Lemma 4.5 .

Proof of Lemma 4.6. The proof is analogous to that of Lemma 4.2 now with $x^{\prime} \in$ $\mathcal{D}_{k}$, upper $k, \delta$-successful if it is upper $k$-successful, and such that

$$
\mu_{T}^{X}\left(D\left(x^{\prime}, \epsilon_{k, j}^{\prime}\right)\right) \leq \Lambda a(1+\delta)\left(\epsilon_{k, j}^{\prime}\right)^{\beta}\left|\log \epsilon_{k, j}^{\prime}\right|, \quad \forall j=0, \ldots, 3 k \log (k+1) .
$$

Here again, the application of Lemma $\left[2.3\right.$ is with $\gamma_{1}=\beta$ and $\gamma_{2}=1$, otherwise using (2.18) and (2.19) as in the proof of Lemma 4.4 The task of completing the details is left to the reader.

\section{The COARSE MULTI-FRACTAL SPECTRUM}

Proof of Proposition 1.5. Fix $a \in(0,1)$ and let

$$
C\left(\varepsilon, a^{2}\right)=\left\{x: \mathcal{I}_{\bar{\theta}, \bar{\theta}^{\prime}}(D(x, \varepsilon)) \geq a^{2} \varepsilon^{2}(\log \varepsilon)^{4}\right\} .
$$

With $\tilde{\varepsilon}_{n}$ as in (2.11), note that

$$
C\left(\tilde{\varepsilon}_{n}, a^{2}\right) \subset \bigcup_{j \in \mathcal{A}_{n}} D\left(x_{j}, \delta \tilde{\varepsilon}_{n}\right) .
$$

Hence, the bound (2.15) yields for some $c_{i}=c_{i}(\delta)<\infty$ and any $\eta>0$,

$$
\begin{aligned}
\mathbf{P}\left(\mathcal{L} e b\left(C\left(\tilde{\varepsilon}_{n}, a^{2}\right)\right) \geq \tilde{\varepsilon}_{n}^{\eta}\right) & \leq \tilde{\varepsilon}_{n}^{-\eta} \mathbb{E}\left(\mathcal{L} e b\left(C\left(\tilde{\varepsilon}_{n}, a^{2}\right)\right)\right. \\
& \leq c_{1} \mathbb{E}\left|\mathcal{A}_{n}\right| \tilde{\varepsilon}_{n}^{2-\eta} \leq c_{2} \tilde{\varepsilon}_{n}^{(1-10 \delta) 2 a-\eta} .
\end{aligned}
$$

The Borel-Cantelli lemma and (2.12) then imply that

$$
\liminf _{\varepsilon \rightarrow 0} \frac{\log \mathcal{L} e b\left(C\left(\varepsilon, a^{2} /(1-\delta)\right)\right)}{\log \varepsilon} \geq 2 a(1-10 \delta), \quad \text { a.s. }
$$

Taking $\delta \rightarrow 0$ then yields the conclusion

$$
\liminf _{\varepsilon \rightarrow 0} \frac{\log \mathcal{L} e b\left(C\left(\varepsilon, a^{2}\right)\right)}{\log \varepsilon} \geq 2 a, \quad \text { a.s. }
$$

Turning to a complementary upper bound, fix $\delta>0$ such that $a^{2}(1+\delta)^{3}<1$. Let $\epsilon_{\delta}=\epsilon \delta /(1+\delta), C_{\delta}=C\left(\varepsilon /(1+\delta), a^{2}(1+\delta)^{3}\right)$ and $N(\epsilon)$ a (finite) maximal set of $x_{i} \in C_{\delta}$ such that $\left|x_{i}-x_{j}\right|>2 \epsilon_{\delta}$ for all $i \neq j$. Note that $\left\{D\left(x_{i}, \epsilon_{\delta}\right): x_{i} \in N(\epsilon)\right\}$ are disjoint and if $x \in C_{\delta}$, then $D\left(x, \epsilon_{\delta}\right) \subset C\left(\varepsilon, a^{2}\right)$. Therefore,

$$
\pi \epsilon_{\delta}^{2}|N(\epsilon)| \leq \mathcal{L} e b\left(\bigcup_{x \in C_{\delta}} D\left(x, \epsilon_{\delta}\right)\right) \leq \mathcal{L} e b\left(C\left(\varepsilon, a^{2}\right)\right) .
$$


With $d(\epsilon)=\log |N(\varepsilon)| / \log (1 / \epsilon)$, we thus see that

$$
\liminf _{\epsilon \rightarrow 0} d(\epsilon) \leq 2-\limsup _{\varepsilon \rightarrow 0} \frac{\log \mathcal{L} e b\left(C\left(\varepsilon, a^{2}\right)\right)}{\log \varepsilon} .
$$

Let

$$
\text { CThicklnt }_{\geq a^{2}}=\left\{x \in D(0,1): \liminf _{\varepsilon \rightarrow 0} \frac{\mathcal{I}_{\bar{\theta}, \bar{\theta}^{\prime}}(D(x, \varepsilon))}{\varepsilon^{2}\left(\log \frac{1}{\varepsilon}\right)^{4}} \geq a^{2}\right\}
$$

and

$$
\text { CThicklnt }_{\gamma, \geq a^{2}}=\left\{x \in D(0,1): \inf _{\epsilon \leq \gamma} \frac{\mathcal{I}_{\bar{\theta}, \bar{\theta}^{\prime}}(D(x, \varepsilon))}{\varepsilon^{2}\left(\log \frac{1}{\varepsilon}\right)^{4}} \geq a^{2}\right\} .
$$

The sets CThickInt ${ }_{\gamma, \geq a^{2}}$ are monotone nonincreasing in $\gamma$ and

$$
\text { CThickInt }_{\geq a^{2}(1+\delta)^{4}} \subseteq \bigcup_{n} \text { CThickInt } \gamma_{\gamma_{n}, \geq a^{2}(1+\delta)^{3}}
$$

for any $\gamma_{n} \rightarrow 0$. Recall that $\mathcal{S}_{\epsilon}:=\left\{D\left(x_{i}, 3 \epsilon_{\delta}\right): x_{i} \in N(\epsilon)\right\}$ forms a cover of $C_{\delta}$; so a fortiori it is also a cover of CThicklnt $\varepsilon_{\varepsilon /(1+\delta), \geq a^{2}(1+\delta)^{3}}$. Fixing $\epsilon_{n} \downarrow 0$ it follows from (5.3) that $\bigcup_{n \geq m} \mathcal{S}_{\epsilon_{n}}$ is a cover of CThickInt $\geq a^{2}(1+\delta)^{4}$ by sets of maximal diameter $6 \epsilon_{m}$. Hence, the $\eta$-Hausdorff measure of CThicklnt $\geq a^{2}(1+\delta)^{4}$ is finite for any $\eta$ such that

$$
\sum_{n=1}^{\infty}\left|N\left(\epsilon_{n}\right)\right| \epsilon_{n}^{\eta}=\sum_{n=1}^{\infty} \epsilon_{n}^{\eta-d\left(\epsilon_{n}\right)}<\infty,
$$

that is, whenever $\eta>\liminf _{\epsilon \rightarrow 0} d(\epsilon)$. Consequently, by (5.1),

$$
\operatorname{dim}\left(\text { CThicklnt }_{\geq a^{2}(1+\delta)^{4}}\right) \leq \liminf _{\epsilon \rightarrow 0} d(\epsilon) \leq 2-\limsup _{\varepsilon \rightarrow 0} \frac{\log \mathcal{L} e b\left(C\left(\varepsilon, a^{2}\right)\right)}{\log \varepsilon} .
$$

Since the set considered in (1.10) is contained in CThicklnt $x_{\geq a^{2}}$, taking $\delta \rightarrow 0$ and using (1.10) yields that

$$
\limsup _{\varepsilon \rightarrow 0} \frac{\log \mathcal{L} e b\left(C\left(\varepsilon, a^{2}\right)\right)}{\log \varepsilon} \leq 2 a, \quad \text { a.s. },
$$

as needed to complete the proof.

\section{Proof of Theorem 1.6: The Upper bound}

Throughout this section, fix $0<r_{1} \leq r$, let $\bar{\theta}_{r}=\inf \left\{s>0:\left|W_{s}\right|=r\right\}$, and define

$$
\overline{\mathcal{I}}^{W, X}=\mathcal{I}_{\bar{\theta}_{r}}^{W, X}\left(D\left(0, r_{1}\right)\right) .
$$

Lemma 6.1. For each $\delta>0$ we can find $c<\infty$ such that for all $k \geq 1, r_{1} \leq$ $r / 2 \leq 1$, and $x_{0}, x_{0}^{\prime}$ with $\left|x_{0}\right|=\left|x_{0}^{\prime}\right|=r_{1}$,

$$
\mathbb{E}^{x_{0}, x_{0}^{\prime}}\left(\overline{\mathcal{I}}^{W, X} / r_{1}^{\beta}\right)^{k} \leq c(k !)^{2}\left((1+\delta) \log \left(r / r_{1}\right)+c\right)^{k} .
$$

Proof of Lemma 6.1. It follows from (1.15) that

$$
\begin{aligned}
& \mathbb{E}^{x_{0}, x_{0}^{\prime}}\left(\left(\overline{\mathcal{I}}^{W, X}\right)^{k}\right) \\
= & k !\left(\pi \Lambda^{-1}\right)^{k} \sum_{\sigma} \int_{D\left(0, r_{1}\right)^{k}} \prod_{j=1}^{k} g_{r}\left(y_{\sigma(j-1)}, y_{\sigma(j)}\right) u^{0}\left(y_{j-1}-y_{j}\right) d y_{j},
\end{aligned}
$$


where the sum runs over all permutations $\sigma$ of $\{1, \ldots, k\}$ and we use the convention that $y_{\sigma(0)}=x_{0}$ and $y_{0}=x_{0}^{\prime}$ (see (2.2) for a similar formula in the case of two Brownian motions).

Recalling (2.3), after scaling in $r_{1}$, (6.2) can be bounded above by

$$
(k !)^{2}\left(\Lambda^{-1}\right)^{k} r_{1}^{k \beta} \sum_{l=0}^{k}\left(\log \left(r / r_{1}\right)+c_{o} \pi\right)^{l} \sum_{|\mathcal{A}|=k-l} J_{\mathcal{A}}
$$

where the sum goes over all subsets $\mathcal{A}$ of $\{1, \ldots, k\}$ of cardinality $k-l$, and

$$
J_{\mathcal{A}}:=\sup _{\sigma,\left|y_{0}\right|=\left|y_{\sigma(0)}\right|=1} \int_{D(0,1)^{k}} \prod_{j \in \mathcal{A}}\left|\log \left(\left|y_{\sigma(j)}-y_{\sigma(j-1)}\right|\right)\right| \prod_{j=1}^{k} u^{0}\left(y_{j-1}-y_{j}\right) d y_{j} .
$$

Fix $p>1$ and $q=p /(p-1)$, and write

$$
\bar{L}_{q}=\max _{m=1,2} \sup _{x \in D(0,1)}\left(\int_{D(0,1)}|\log (|y-x|)|^{m q} d y\right)^{1 / m q} .
$$

Let $\Lambda_{p}$ denote the $L^{2}(D(0,1), d x)$ norm of the operator $K^{(p)} f(x)=\int_{D(0,1)} u^{0}(x-$ $y)^{p} f(y) d y$ with kernel $\left(u^{0}\right)^{p}$. Note first that for some fixed $r$, we have that

$$
C_{p}:=\sup _{\left|y_{0}\right|=1,\|f\|_{2} \leq 1}\left|\left(K^{(p)}\right)^{r} f(y)\right|<\infty .
$$

Noting that each variable $y_{j}$ appears in at most a pair of logarithmic factors, we have, using Hölder's inequality, that

$$
J_{\mathcal{A}} \leq \bar{L}_{q}^{k-l} \pi^{l / q}\left(C_{p} \Lambda_{p}^{(k-r)} \pi^{1 / 2}\right)^{1 / p} .
$$

Hence, by (6.3), we have that for some finite $C_{p}^{\prime}$,

$$
\begin{aligned}
& \mathbb{E}^{x_{0}, x_{0}^{\prime}}\left(\left(\overline{\mathcal{I}}^{W, X}\right)^{k}\right) \\
& \quad \leq C_{p}^{\prime}(k !)^{2}\left(\frac{\Lambda_{p}^{1 / p}}{\Lambda}\right)^{k} r_{1}^{k \beta} \sum_{l=0}^{k}\left(\pi^{1 / q} \log \left(r / r_{1}\right)+\pi^{1+1 / q} c_{o}\right)^{l}\left(\begin{array}{l}
k \\
l
\end{array}\right) \bar{L}_{q}^{k-l} .
\end{aligned}
$$

Now taking $p$ large enough such that $\Lambda_{p}^{1 / p} \pi^{1 / q}<(1+\delta) \Lambda$ (which is possible since for $p>1$ small enough, $\Lambda_{p}<\infty$, and using interpolation, $\Lambda_{p} \rightarrow_{p \rightarrow 1} \Lambda$ ), we get (6.1) with $c=\left(c_{o} \pi^{1+1 / q}+\bar{L}_{q}\right) \vee C_{p}^{\prime}$.

The next lemma follows by the same arguments as in the proof of Lemma 2.2 now with $F=\sqrt{4 \mathcal{I}_{\bar{\theta}_{2}}^{W, X^{\prime}}(D(0, \varepsilon)) /\left(\varepsilon^{\beta}|\log \varepsilon|(1+4 \delta)\right)}$.

Lemma 6.2. For any $\delta>0$ we can find $c, y_{0}<\infty$ and $\varepsilon_{0}>0$ so that for all $\varepsilon \leq \varepsilon_{0}$ and $y \geq y_{0}$,

$$
P^{x_{0}, x_{0}^{\prime}}\left(\mathcal{I}_{\bar{\theta}_{2}}^{W, X}(D(0, \varepsilon)) \geq y^{2} \epsilon^{\beta}|\log \epsilon|\right) \leq c \exp (-(1-\delta) 2 y)
$$

for all $x_{0}, x_{0}^{\prime}$ with $\left|x_{0}\right|=\left|x_{0}^{\prime}\right|=\varepsilon$.

The upper bound in Theorem [1.6 is now derived via the same line of reasoning used in [4] (following [2] Section 5]). The details are omitted. 


\section{Proof of Theorem 1.2}

Throughout this section, fix $0<r_{1} \leq r$, let $\bar{\theta}_{r}=\inf \left\{s>0:\left|W_{s}\right|=r\right\}$, and define

$$
\bar{\mu}=\frac{\mu_{\bar{\theta}_{r}}^{W}\left(K\left(0, r_{1}\right)\right)}{|K| / \pi} .
$$

Lemma 7.1. We can find $c<\infty$ such that for all $k \geq 1, r_{1} \leq r_{2} \leq r / 2 \leq 1$, and $x_{0}$ with $\left|x_{0}\right|=r_{2}$,

$$
\mathbb{E}^{x_{0}}\left(\bar{\mu} / r_{1}^{2}\right)^{k} \leq k !\left(\log \left(r / r_{1}\right)+c\right)^{k}
$$

Furthermore,

$$
\mathbb{E}^{x_{0}}\left(\bar{\mu} / r_{1}^{2}\right)=\left(\log \left(r / r_{2}\right) \pm c\right) .
$$

Proof of Lemma 7.1. Let $g_{r}(x, y)$ denote Green's function for $D(0, r)$, i.e., the 0potential density for planar Brownian motion is killed when it first hits $D(0, r)^{c}$. We have

$$
\mathbb{E}^{x_{0}}\left(\bar{\mu}^{k}\right)=k !\left(\frac{\pi}{|K|}\right)^{k} \int_{K\left(0, r_{1}\right)^{k}} \prod_{j=1}^{k} g_{r}\left(y_{j-1}, y_{j}\right) d y_{j}
$$

where we use the convention that $y_{\sigma(0)}=x_{0}$.

Thus, to prove (7.2) we must show that

$$
\frac{\pi}{|K|} \int_{K\left(0, r_{1}\right)} g_{r}\left(x_{0}, y\right) d y=\left(\log \left(r / r_{2}\right) \pm c\right) r_{1}^{2}
$$

for all $x_{0}$ with $\left|x_{0}\right|=r_{2}$. We will also show that

$$
\sup _{x \in K\left(0, r_{1}\right)} \frac{\pi}{|K|} \int_{K\left(0, r_{1}\right)} g_{r}(x, y) d y \leq\left(\log \left(r / r_{1}\right)+c\right) r_{1}^{2} .
$$

If we use this inductively for the $d y_{k}, d y_{k-1}, \ldots, d y_{2}$ integrals in (7.3), and then use (7.4) for the $d y_{1}$ integral, we will establish (7.1). It follows from (2.3) that the left-hand side in (7.5) is bounded by

$$
\begin{aligned}
& \sup _{x \in K\left(0, r_{1}\right)} \frac{1}{|K|} \int_{K\left(0, r_{1}\right)}\left(\log \left(r / r_{1}\right)+\log \left(\frac{r_{1}}{|x-y|}\right)+\pi c_{o}\right) d y \\
& =\left(\log \left(r / r_{1}\right)+\pi c_{o}\right) r_{1}^{2}+r_{1}^{2} \sup _{x \in K} \frac{1}{|K|} \int_{K} \log \left(\frac{1}{|x-y|}\right) d y,
\end{aligned}
$$

and (7.5) follows.

Turning to (7.4), as above, we can write the left-hand side as

$$
\begin{aligned}
& \frac{1}{|K|} \int_{K\left(0, r_{1}\right)}\left(\log \left(r / r_{2}\right)+\log \left(\frac{r_{2}}{\left|x_{0}-y\right|}\right) \pm \pi c_{o}\right) d y \\
= & \left(\log \left(r / r_{2}\right) \pm \pi c_{o}\right) r_{1}^{2}+\frac{1}{|K|} \int_{K\left(0, r_{1}\right)} \log \left(\frac{r_{2}}{\left|x_{0}-y\right|}\right) d y .
\end{aligned}
$$

When $r_{1} / r_{2} \leq 1 / 2$, we have that $\left|\log \frac{r_{2}}{\left|x_{0}-y\right|}\right|$ is bounded on $K\left(0, r_{1}\right)$ so that (17.4) follows in that case. When $1 / 2 \leq r_{1} / r_{2} \leq 1$, we can use

$$
\int_{K\left(0, r_{1}\right)}\left|\log \left(\frac{r_{2}}{\left|x_{0}-y\right|}\right)\right| d y \leq \int_{D\left(0, r_{2}\right)}\left|\log \left(\frac{r_{2}}{\left|x_{0}-y\right|}\right)\right| d y
$$


and (7.4) follows as above.

Continuing as in the proof of Lemma [2.2, now with $F=\bar{\mu} /\left\{r_{1}^{2}\left|\log r_{1}\right|(1+\delta)\right\}$, this easily implies:

Lemma 7.2. For any $\delta>0$ we can find $c, y_{0}<\infty$ and $\varepsilon_{0}>0$ so that for all $\varepsilon \leq \varepsilon_{0}$ and $y \geq y_{0}$,

$$
P^{x_{0}}\left(\mu_{\bar{\theta}_{2}}^{W}(K(0, \varepsilon)) \geq(|K| / \pi) y \epsilon^{2}|\log \epsilon|\right) \leq c \exp (-(1-\delta) y)
$$

for all $x_{0}$ with $\left|x_{0}\right|=\varepsilon$.

We first turn to the proof of the upper bounds. The proof requires a slight adaptation of the technique of [5. Section 2], because it is not true in general that $\epsilon K \subset \epsilon^{\prime} K$ if $\epsilon<\epsilon^{\prime}$.

Let $r_{n}=(1-\delta)^{n}$, and let $\left\{Q_{i, n}\right\}$ be a tiling of $[-1,1]^{2}$ by squares of side $r_{n}$, and let $Q_{i, n}^{j}=j+Q_{i, n}$ where $j=\left(j_{1}, j_{2}\right)$ and $j_{i} \in\left\{0, r_{n} / M, \ldots,(M-1) r_{n} / M\right\}$ with $M=2 / \delta$. We first have the following immediate corollary of Lemma 7.2

Corollary 7.3. There exists an $n_{0}=n_{0}(\omega, \delta)$ such that for all $n>n_{0}$,

$$
\#\left\{(i, j): \mu_{\bar{\theta}}^{W}\left(Q_{i, n}^{j}\right) \geq \frac{a r_{n}^{2}\left|\log r_{n}\right|^{2}}{\pi}\right\} \leq r_{n}^{a(1-\delta)-2} .
$$

Our use of Corollary 7.3 is as follows. By the assumption on the boundary of $K,|K|=|\operatorname{cl} K|$, and hence it is enough to prove the upper bounds for compact $K$. Thus, let $K \subset D(0,1)$ be compact. Fix $\delta>0$. Cover $K$ by a finite number (say $k$ ) of squares $Q_{i}$, with $\sum_{i=1}^{k}\left|Q_{i}\right| \leq|K|(1+\delta / 2)$. Note that $K(x, \epsilon) \subset \bigcup_{i=1}^{k}\left(x+\epsilon Q_{i}\right)$, and hence

$$
\mu_{\bar{\theta}}^{W}(K(x, \epsilon)) \leq \sum_{i=1}^{k} \mu_{\bar{\theta}}^{W}\left(x+\epsilon Q_{i}\right)
$$

Hence,

$$
\left\{x: \limsup _{\epsilon \rightarrow 0} \frac{\pi \mu_{\bar{\theta}}^{W}(K(x, \epsilon))}{|K| \epsilon^{2}|\log \epsilon|^{2}} \geq a(1+\delta / 2)\right\} \subset \bigcup_{i=1}^{k}\left\{x: \limsup _{\epsilon \rightarrow 0} \frac{\pi \mu_{\bar{\theta}}^{W}\left(Q_{i}(x, \epsilon)\right)}{\left|Q_{i}\right| \epsilon^{2}|\log \epsilon|^{2}} \geq a\right\} .
$$

Hence, it is enough to prove the upper bounds on the dimension for an arbitrary square $Q$ of side $b$. Note however that then, with $r_{n+1} \leq \epsilon b \leq r_{n}$, and any $x$, there exist $i, j$ such that $Q(x, \epsilon) \subset Q_{i, n-1}^{j}$, whereas

$$
\left\{x: \frac{\pi \mu_{\bar{\theta}}^{W}(Q(x, \epsilon))}{|Q| \epsilon^{2}|\log \epsilon|^{2}} \geq a\right\} \subset\left\{\bigcup_{i, j} Q_{i, n-1}^{j}: \frac{\pi \mu_{\bar{\theta}}^{W}\left(Q_{i, n-1}^{j}\right)}{|Q| \epsilon^{2}|\log \epsilon|^{2}} \geq a\right\} .
$$

Given Corollary [7.3] and using that $r_{n+1} / r_{n-1}=(1-\delta)^{2}$, one gets immediately that

$$
\operatorname{dim}\left\{x: \limsup _{\epsilon \rightarrow 0} \frac{\pi \mu \bar{\theta}\left(Q_{i}(x, \epsilon)\right)}{\left|Q_{i}\right| \epsilon^{2}|\log \epsilon|^{2}} \geq a\right\} \leq 2-a(1-\delta)^{3}+2 \delta, \text { a.s. }
$$

which yields the required upper bound on the dimension. The estimate (1.6) is similarly proved.

Surprisingly, the lower bounds in Theorem 1.2 require no further computations. First, we note the following slight adaptation of [5, Lemma 3.1]. 
Lemma 7.4. Let $Q \subset D(0,1)$ be a fixed square, not necessarily centered. Then there exist $\delta(\epsilon)=\delta(\epsilon, \omega, Q) \rightarrow 0$ a.s. such that for all $m$ and all $x \in D(0,1 / 2) \backslash$ $D(0,1 / 8)$, if $x$ is m-perfect, then

$$
a-\delta(\epsilon) \leq \frac{\pi \mu_{\bar{\theta}}^{W}(Q(x, \epsilon))}{\epsilon^{2}(\log \epsilon)^{2}|Q|} \leq a+\delta(\epsilon), \forall \epsilon \geq \epsilon_{m}
$$

Proof of Lemma 7.4. Simply follow the arguments of [5, Section 6] using Lemma 7.1] wherever [5. Lemma 2.1] is used there.

Let

$$
E=\left\{x \in D(0,1 / 2) \backslash D(0,1 / 8): \exists x_{n} \rightarrow x \text { such that } x_{n} \text { is } n \text {-perfect }\right\} .
$$

Recall that

$$
\operatorname{dim}(E)=2-a, \text { a.s. }
$$

It follows from Lemma 7.4 that for any fixed square $Q$, a.s.

$$
\limsup _{\epsilon \rightarrow 0} \frac{\pi \mu_{\bar{\theta}}^{W}(Q(x, \epsilon))}{\epsilon^{2}(\log \epsilon)^{2}|Q|}=a, \quad \forall x \in E .
$$

Consequently, for any compact $F \subset D(0,1)$, a.s.

$$
\limsup _{\epsilon \rightarrow 0} \frac{\pi \mu_{\bar{\theta}}^{W}(F(x, \epsilon))}{\epsilon^{2}(\log \epsilon)^{2}|F|} \leq a, \quad \forall x \in E .
$$

(Just cover $F$ by a finite number of squares $Q_{i} \subset D(0,1)$ with $\sum_{i}\left|Q_{i}\right| \leq|F|(1+\delta)$, considering first the $\limsup$ as $\epsilon \rightarrow 0$, then taking $\delta \rightarrow 0$.) For $K$ satisfying the assumptions of the theorem we have from the above that

$$
\limsup _{\epsilon \rightarrow 0} \frac{\pi \mu_{\bar{\theta}}^{W}(K(x, \epsilon))}{\epsilon^{2}(\log \epsilon)^{2}|K|} \leq \limsup _{\epsilon \rightarrow 0} \frac{\pi \mu_{\bar{\theta}}^{W}(\operatorname{cl} K(x, \epsilon))}{\epsilon^{2}(\log \epsilon)^{2}|K|} \leq a, \quad \forall x \in E .
$$

Recall that, for $F=\operatorname{cl}(D(0,1) \backslash K)$ and all $x$,

$$
\begin{aligned}
\mu_{\bar{\theta}}^{W}(K(x, \epsilon)) & =\mu_{\bar{\theta}}^{W}(D(x, \epsilon))-\mu_{\bar{\theta}}^{W}(D(x, \epsilon) \backslash K(x, \epsilon)) \\
& \geq \mu_{\bar{\theta}}^{W}(D(x, \epsilon))-\mu_{\bar{\theta}}^{W}(F(x, \epsilon)),
\end{aligned}
$$

whereas by [5, Lemma 3.1], a.s.

$$
\lim _{\epsilon \rightarrow 0} \frac{\mu_{\bar{\theta}}^{W}(D(x, \epsilon))}{\epsilon^{2}(\log \epsilon)^{2}}=a, \quad \forall x \in E .
$$

Noting that $|F|=|D(0,1) \backslash K|$ by our assumption on the boundary of $K$, and using (7.7), we conclude that a.s.

$$
\liminf _{\epsilon \rightarrow 0} \frac{\mu_{\bar{\theta}}^{W}(K(x, \epsilon))}{\epsilon^{2}(\log \epsilon)^{2}} \geq a-\frac{a|D(0,1) \backslash K|}{\pi}=\frac{a|K|}{\pi}, \quad \forall x \in E .
$$

When combined with (7.8), this implies that a.s.

$$
\lim _{\epsilon \rightarrow 0} \frac{\pi \mu_{\bar{\theta}}^{W}(K(x, \epsilon))}{\epsilon^{2}(\log \epsilon)^{2}|K|}=a, \quad \forall x \in E ;
$$

so (7.6) yields the required lower bound on the dimension of the sets in (1.7). 


\section{Proof of Theorem 1.1}

Recall the local times

$$
L_{n}(x):=\sum_{k=0}^{n} \mathbf{1}_{\left\{X_{k}=x\right\}}, \quad L_{n}^{\prime}(x):=\sum_{k=0}^{n} \mathbf{1}_{\left\{X_{k}^{\prime}=x\right\}} \quad\left(x \in \mathbf{Z}^{2}\right)
$$

of the simple random walks $\left\{X_{k}\right\}$ and $\left\{X_{k}^{\prime}\right\}$ and let $L_{n}^{X, X^{\prime}}(x)=L_{n}^{X}(x) L_{n}^{X^{\prime}}(x)$. We next compute upper bounds on $M_{n}^{X, X^{\prime}}(b)=\left|\left\{x \in \mathbf{Z}^{2}: L_{n}^{X}(x) L_{n}^{X^{\prime}}(x) \geq b\right\}\right|$ and $T_{n}^{X, X^{\prime}}$. To this end, using the Markov property and translation invariance of SRW in $\mathbf{Z}^{2}$, as well as the bound of [5. (5.11)], we have for any $\delta>0$, all $n \geq n_{0}(\delta)$, $\alpha>0$ and $x \in \mathbf{Z}^{2}$,

$$
\mathbf{P}\left[L_{n}(x) \geq \alpha(\log n)^{2}\right] \leq \mathbf{P}\left[L_{n}(0) \geq \alpha(\log n)^{2}\right] \leq n^{-(1-\delta) \pi \alpha} .
$$

Thus, fixing $0<\delta<b$ and $K>(2 b-\delta) / \delta$, by the independence of $\left\{X_{k}\right\}$ and $\left\{X_{k}^{\prime}\right\}$, we have for $n \geq n_{0}(\delta)$ and all $x \in \mathbf{Z}^{2}$,

$$
\begin{aligned}
& \mathbf{P}\left(L_{n}^{X, X^{\prime}}(x) \geq b^{2}(\log n)^{4}\right) \\
& \quad \leq \sum_{i=1}^{K} \mathbf{P}\left[\frac{L_{n}(x)}{(\log n)^{2}} \geq(i-1) \delta, \frac{L_{n}^{\prime}(x)}{(\log n)^{2}} \geq \frac{b^{2}}{i \delta}\right]+\mathbf{P}\left[\frac{L_{n}(x)}{(\log n)^{2}} \geq K \delta\right] \\
& \quad \leq \sum_{i=1}^{K} n^{-(1-\delta) \pi\left((i-1) \delta+b^{2} /(i \delta)\right)}+n^{-(1-\delta) \pi K \delta} \leq 2 K n^{-(1-\delta) \pi(2 b-\delta)}
\end{aligned}
$$

(as $s+b^{2} / s \geq 2 b$ for all $s>0$ ).

Let $R_{n}=\max _{k \leq n}\left(\left|X_{k}\right| \vee\left|X_{k}^{\prime}\right|\right)$, noting that for some $c>0$ and all $n$ large,

$$
\mathbf{P}\left(R_{n} \geq n^{1 / 2+\delta}\right) \leq e^{-c n^{\delta}} .
$$

Fixing $0<\delta<b$, set $\gamma=1+4 \delta-(1-\delta) \pi(2 b-\delta)$. Then, by (8.1) and (8.2), we have for all $n$ large enough,

$$
\begin{aligned}
& \mathbf{P}\left(M_{n}^{X, X^{\prime}}\left(b^{2}(\log n)^{4}\right) \geq n^{\gamma}\right) \\
& \quad \leq \mathbf{P}\left(R_{n} \geq n^{1 / 2+\delta}\right)+n^{-\gamma} \mathbb{E}\left(M_{n}^{X, X^{\prime}}\left(b^{2}(\log n)^{4}\right) ; R_{n}<n^{1 / 2+\delta}\right) \\
& \leq e^{-c n^{\delta}}+n^{-\gamma} \sum_{|x| \leq n^{1 / 2+\delta}} \mathbf{P}\left(L_{n}^{X, X^{\prime}}(x) \geq b^{2}(\log n)^{4}\right) \leq n^{-\delta} .
\end{aligned}
$$

For $b<1 /(2 \pi)$, taking $\delta \downarrow 0$, it follows by $(\underline{8.3})$ and the Borel-Cantelli lemma that almost surely

$$
\limsup _{m \rightarrow \infty} \frac{\log M_{n_{m}}^{X, X^{\prime}}\left(b^{2}\left(\log n_{m}\right)^{4}\right)}{\log n_{m}} \leq 1-2 \pi b
$$

on the subsequence $n_{m}=2^{m}$. By the monotonicity of $n \mapsto \log L_{n}^{X, X^{\prime}}(x)$ and $n \mapsto \log n$, one easily checks that (8.4) holds also when replacing $n_{m}$ with $m$, yielding the upper bound in (1.4). In case $b>1 /(2 \pi)$ we note that $\gamma=\gamma(\delta)<0$ when $\delta>0$ is small enough; so (8.3) implies that for all $n$ large enough,

$$
\mathbf{P}\left(T_{n}^{X, X^{\prime}} \geq b^{2}(\log n)^{4}\right)=\mathbf{P}\left(M_{n}^{X, X^{\prime}}\left(b^{2}(\log n)^{4}\right) \geq 1\right) \leq n^{-\delta} .
$$


Therefore, taking $b \downarrow 1 /(2 \pi)$, it follows by the Borel-Cantelli lemma that almost surely,

$$
\limsup _{m \rightarrow \infty} \frac{T_{n_{m}}^{X, X^{\prime}}}{\left(\log n_{m}\right)^{4}} \leq \frac{1}{4 \pi^{2}}
$$

on the subsequence $n_{m}=2^{m}$. The monotonicity of $n \mapsto T_{n}^{X, X^{\prime}}$ and $n \mapsto(\log n)^{4}$ allows us to replace $n_{m}$ with $m$, leading to the upper bound of (1.3).

It suffices to prove the complementary lower bounds for (1.4), because the lower bound in (1.3) then directly follows. As in [5], the proof uses the strong approximation results of [6].

For any $A, A^{\prime} \subseteq \mathbf{N}$ let

$$
\begin{gathered}
L_{A \times A^{\prime}}^{X, X^{\prime}}(z)=\#\left\{(i, j) \in A \times A^{\prime}: X_{i}=X_{j}^{\prime}=z\right\}, \\
M\left\{A \times A^{\prime}, b\right\}=\#\left\{z \in \mathbf{Z}^{2}: L_{A \times A^{\prime}}^{X, X^{\prime}}(z) \geq b\right\},
\end{gathered}
$$

so that $M_{n}^{X, X^{\prime}}(b)=M\left\{[0, n]^{2}, b\right\}$. Let $n_{j, i}=j^{8 i} e^{j^{2}}$ and $\Delta n_{j, i}=n_{j, i}-n_{j, i-1}$. Fix $\epsilon>0$. We claim that there exists some $j_{0}=j_{0}(\omega)<\infty$ a.s. such that for all $j \geq j_{0}$,

$$
\max _{1 \leq i \leq j} M\left\{\left[n_{j, i-1}, n_{j, i}\right]^{2}, b^{2}\left(\log \Delta n_{j, i}\right)^{4}\right\} \geq\left(\Delta n_{j, i}\right)^{1-2 \pi b-\epsilon} .
$$

Assuming this for the moment, we see from (8.7) that for any $n_{j, j} \leq n \leq n_{j+1, j+1}$ with $j$ sufficiently large,

$$
M\left\{[0, n]^{2},(1-\epsilon)^{2} b^{2}(\log n)^{4}\right\} \geq n^{(1-\epsilon)^{2}(1-2 \pi b-\epsilon)},
$$

so that, replacing $b$ by $b /(1-\epsilon)$, we have

$$
\liminf _{n \rightarrow \infty} \frac{\log M_{n}^{X, X^{\prime}}\left(b^{2}(\log n)^{4}\right)}{\log n} \geq(1-\epsilon)^{2}\left(1-\frac{2 \pi b}{1-\epsilon}-\epsilon\right), \quad \text { a.s. }
$$

and taking $\epsilon \rightarrow 0$ completes the proof of the lower bound of Theorem 1.1 subject only to (8.7) which we now establish.

Note that with $\Psi_{j, i}=\left\{\left|X_{n_{j, i-1}}\right| \vee\left|X_{n_{j, i-1}}^{\prime}\right| \leq \sqrt{\Delta n_{j, i}} / \log \Delta n_{j, i}\right\}$ we have

$$
\begin{gathered}
\mathbf{P}\left(\max _{1 \leq i \leq j} M\left\{\left[n_{j, i-1}, n_{j, i}\right]^{2}, b^{2}\left(\log \Delta n_{j, i}\right)^{4}\right\} \leq\left(\Delta n_{j, i}\right)^{1-2 \pi b-\epsilon}\right) \\
\leq j \max _{1 \leq i \leq j} \mathbf{P}\left(\Psi_{j, i}^{c}\right) \\
+\mathbf{P}\left(\bigcap_{1 \leq i \leq j}\left\{M\left\{\left[n_{j, i-1}, n_{j, i}\right]^{2}, b^{2}\left(\log \Delta n_{j, i}\right)^{4}\right\} \leq\left(\Delta n_{j, i}\right)^{1-2 \pi b-\epsilon} ; \Psi_{j, i}\right\}\right) .
\end{gathered}
$$

We will show that this is summable in $j$ so that (8.7) will follow by the BorelCantelli Lemma. By [18, Theorem 17.5] (which is essentially the Central Limit Theorem), for some $c>0$ and all $j$ sufficiently large,

$$
\mathbf{P}\left(\left|X_{n_{j, i-1}}\right|>\sqrt{\Delta n_{j, i}} / \log \Delta n_{j, i}\right) \leq \mathbf{P}\left(\frac{\left|X_{n_{j, i-1}}\right|}{\sqrt{n_{j, i-1}}}>j\right) \leq e^{-c j},
$$


so that the first term on the right-hand side of (8.10) is summable in $j$. On the other hand, since

$$
\begin{aligned}
& M\left\{\left[n_{j, i-1}, n_{j, i}\right]^{2}, b^{2}\left(\log \Delta n_{j, i}\right)^{4}\right\} \\
& =M\left\{\left[0, \Delta n_{j, i}\right]^{2}, b^{2}\left(\log \Delta n_{j, i}\right)^{4}\right\} \circ\left(\theta_{n_{j, i-1}}, \theta_{n_{j, i-1}}^{\prime}\right),
\end{aligned}
$$

the Markov property together with the following lemma will bound the second term on the right-hand side of (8.10) by $\left(1-p_{0}\right)^{j}$, which is also summable in $j$. Thus, with $D_{0}(r)=D(0, r) \cap \mathbf{Z}^{2}$, (8.7) and consequently the proof of the theorem is reduced to the following lemma.

Lemma 8.1. For any $\epsilon>0$ we can find $p_{0}>0$ such that for all $n$ sufficiently large and all $x, x^{\prime} \in D_{0}(\sqrt{n} / \log n)$,

$$
\mathbf{P}^{x} \times \mathbf{P}^{\prime x^{\prime}}\left(M_{n}^{X, X^{\prime}}\left(b^{2}(\log n)^{4}\right) \geq n^{1-2 \pi b-\epsilon}\right) \geq p_{0}>0 .
$$

Proof of Lemma 8.1. For any $B \subseteq \mathbf{Z}^{2}$, let

$$
M\left\{A \times A^{\prime}, B, b\right\}=\#\left\{z \in B: L_{A \times A^{\prime}}^{X, X^{\prime}}(z) \geq b\right\}
$$

and note that if $\tau_{B}=\inf \left\{i \geq 0: X_{i} \in B\right\}, \tau_{B}^{\prime}=\inf \left\{i \geq 0: X_{i}^{\prime} \in B\right\}$, we have

$$
M\left\{A \times A^{\prime}, B, b\right\}=M\left\{\left(A-\tau_{B}\right) \times\left(A^{\prime}-\tau_{B}^{\prime}\right), B, b\right\} \circ\left(\theta_{\tau_{B}}, \theta_{\tau_{B}^{\prime}}^{\prime}\right) .
$$

We will use the abbreviation $\widetilde{b}=1-2 \pi b$. Let $\tau_{n}=\tau_{D_{0}^{c}(2 \sqrt{n} / \log n)}$ and $\tau_{n}^{\prime}=$ $\tau_{D_{0}^{c}(2 \sqrt{n} / \log n)}^{\prime}$. By $(\underline{8.14})$ and the Markov property,

$$
\begin{gathered}
\mathbf{P} \times \mathbf{P}^{\prime}\left(M\left\{[0, n]^{2}, D_{0}^{c}(2 \sqrt{n} / \log n), b^{2}(\log n)^{4}\right\} \geq n^{\widetilde{b}-\epsilon}\right) \\
\leq \mathbb{E} \times \mathbb{E}^{\prime}\left\{\mathbf{P}^{X_{\tau_{n}}} \times \mathbf{P}^{\prime X_{\tau_{n}}^{\prime}}\left(M \left\{[0, n]^{2}, D_{0}^{c}(2 \sqrt{n} / \log n),\right.\right.\right. \\
\left.\left.\left.b^{2}(\log n)^{4}\right\} \geq n^{\widetilde{b}-\epsilon}\right)\right\} \\
=\sum_{y, y^{\prime}} H_{n}(0, y) H_{n}\left(0, y^{\prime}\right) \mathbf{P}^{y} \times \mathbf{P}^{y^{\prime}}\left(M \left\{[0, n]^{2}, D_{0}^{c}(2 \sqrt{n} / \log n),\right.\right. \\
\left.\left.b^{2}(\log n)^{4}\right\} \geq n^{\widetilde{b}-\epsilon}\right)
\end{gathered}
$$

where $H_{n}(x, y)=\mathbf{P}^{x}\left(X_{\tau_{n}}=y\right)$ is a harmonic measure. By Harnack's inequality [13, Theorem 1.7.2], for some $C<\infty$ and all $n, y$,

$$
H_{n}(0, y) \leq C \inf _{x \in D_{0}(\sqrt{n} / \log n)} H_{n}(x, y) .
$$

Using (8.14) and the Markov property again shows that uniformly in $x, x^{\prime} \in$ $D_{0}(\sqrt{n} / \log n)$,

$$
\begin{aligned}
\mathbf{P} \times \mathbf{P}^{\prime} & \left(M\left\{[0, n]^{2}, D_{0}^{c}(2 \sqrt{n} / \log n), b^{2}(\log n)^{4}\right\} \geq n^{\widetilde{b}-\epsilon}\right) \\
\leq & C \mathbf{P}^{x} \times \mathbf{P}^{\prime x^{\prime}}\left(M\left\{\left[0, n+\tau_{n}\right] \times\left[0, n+\tau_{n}^{\prime}\right], b^{2}(\log n)^{4}\right\} \geq n^{\widetilde{b}-\epsilon}\right) \\
\leq & C \mathbf{P}^{x} \times \mathbf{P}^{\prime x^{\prime}}\left(M\left\{[0,(1+\epsilon) n]^{2}, b^{2}(\log n)^{4}\right\} \geq n^{\widetilde{b}-\epsilon}\right) \\
& +C \mathbf{P}^{x}\left(\tau_{n}>\epsilon n\right)+C \mathbf{P}^{x^{\prime}}\left(\tau_{n}^{\prime}>\epsilon n\right) .
\end{aligned}
$$

Since $\mathbf{P}^{x}\left(\tau_{n}>\epsilon n\right)=\mathbf{P}^{x}\left(\max _{j \leq \epsilon n}\left|X_{j}\right|<2 \sqrt{n} / \log n\right)$, we see from the last line of [18, Theorem 17.5] that for $n$ sufficiently large, the last line of (8.17) is negligible, 
so that given the next lemma, we obtain Lemma 8.1 after some adjustment of $b$ and $\epsilon$.

Lemma 8.2. For any $\epsilon>0$ we can find $p_{0}>0$ such that for all $n$ sufficiently large,

$$
\mathbf{P} \times \mathbf{P}^{\prime}\left(M\left\{[0, n]^{2}, D_{0}^{c}(2 \sqrt{n} / \log n), b^{2}(\log n)^{4}\right\} \geq n^{1-2 \pi b-3 \epsilon}\right) \geq p_{0} .
$$

Proof of Lemma 8.2. This lemma is derived from results about Brownian motion by using strong approximation. However, for $M\left\{[0, n]^{2}, D_{0}^{c}(2 \sqrt{n} / \log n), b^{2}(\log n)^{4}\right\}$, results are not easy to obtain directly by strong approximation since $L_{[0, n]^{2}}^{X, X^{\prime}}(z)$ does not correspond to a functional of Brownian motion. Instead, we will derive results for $L_{[0, n]^{2}}^{X, X^{\prime}}(z)$ from results about excursions of random walks between concentric discs, and it is such results which can be obtained from our work on Brownian thick points. To this end we introduce notation which is meant to simplify the connection with Brownian motion.

Fix $a<1$ and $\delta>0$ small. Set $m_{k}=a k^{2}$ and $k(n)=[(1 / 2-\delta) \log n]$ so that $e^{-k(n)} \geq n^{\delta-1 / 2}$. Let $D_{z}(r)=D(z, r) \cap \mathbf{Z}^{2}$ denote the disc of radius $r$ in $\mathbf{Z}^{2}$ centered at $z$ and define its boundary $\partial D_{z}(r)=\left\{z^{\prime} \notin D_{z}(r):\left|z^{\prime}-y\right|=\right.$ 1 for some $\left.y \in D_{z}(r)\right\}$. For any fixed $K<\infty$, let

$$
r_{n}=(1+3 \delta) e^{-k(n)} \sqrt{n /(2 K)}, \quad R_{n}=(1-3 \delta) e^{-k(n)+1} \sqrt{n /(2 K)} .
$$

We say that $z \in \mathbf{Z}^{2}$ is $n, \delta$-admissible if at least $(1-2 \delta) m_{k(n)}$ excursions between $\partial D_{z}\left(r_{n}\right)$ and $\partial D_{z}\left(R_{n}\right)$ are completed by both $\left\{X_{i}\right\}$ and $\left\{X_{i}^{\prime}\right\}$ for $i \leq n$. Let

$$
I_{n, \delta}:=\left\{z \in D_{0}(\sqrt{n /(2 K)}) \cap D_{0}^{c}(2 \sqrt{n} / \log n): z \text { is } n, \delta \text {-admissible }\right\} .
$$

Lemma 8.2 now follows from the next lemma if we take $a=2 \pi b /(1-\epsilon)$ and $\epsilon, \delta$ sufficiently small.

Lemma 8.3. For any $\epsilon>0$ we can find $K_{0}<\infty$ and $\delta_{0}>0$ such that for all $K>K_{0}$ and $\delta<\delta_{0}$

$$
\liminf _{n \rightarrow \infty} \mathbf{P} \times \mathbf{P}^{\prime}\left(\left|I_{n, \delta}\right| \geq n^{1-a-\epsilon}\right) \geq 1 / 2
$$

and

$$
\lim _{n \rightarrow \infty} \mathbf{P} \times \mathbf{P}^{\prime}\left(\inf _{x \in I_{n, \delta}} L_{n}^{X, X^{\prime}}(x) \leq \frac{(1-\epsilon)^{2} a^{2}}{4 \pi^{2}}(\log n)^{4}\right)=0 .
$$

Proof of Lemma 8.3. We first prove (8.20). Fixing $n$, let $\sigma_{z}$ denote the time it takes $\left\{X_{i}\right\}$ to complete $(1-2 \delta) m_{k(n)}$ excursions between $\partial D_{z}\left(r_{n}\right)$ and $\partial D_{z}\left(R_{n}\right)$, after first hitting $\partial D_{z}\left(R_{n}\right)$, and denote by $Y_{j}(z)$ the occupation measure of $z$ by $\left\{X_{i}\right\}$ during its $j$ th such excursion. Thus, fixing the starting point of the $j$ th excursion, $Y_{j}(z)$ is distributed as $L_{T_{R_{n}}}(0)$ where $T_{R_{n}}$ is the first hitting time of $\partial D_{0}\left(R_{n}\right)$ (when starting at the corresponding $\left.z^{\prime} \in \partial D_{0}\left(r_{n}\right)\right)$. We note that

$$
\mathbb{E}^{0}\left(\left\{L_{T_{R_{n}}}(0)\right\}^{k}\right)=k !\left\{G_{R_{n}}(0,0)\right\}^{k} \quad k=1,2, \ldots
$$

where $G_{R_{n}}(x, y)$ is Green's function for $D_{0}\left(R_{n}\right)$. This is a simple case of Kac's moment formula; see [7]. We will only need $k=1$ and 2 . We will use the abbreviation $G_{n}=G_{R_{n}}(0,0)$. 
Set $\lambda=\delta^{1 / 4}, \ell_{n}=(1-2 \lambda)^{2} /(\delta \log n)$. Since

$$
L_{\sigma_{z}}(z) \geq \sum_{j=1}^{(1-2 \delta) m_{k(n)}} Y_{j}(z),
$$

it follows by the strong Markov property of $\left\{X_{i}\right\}$ at the start of each of these excursions that

$$
\begin{aligned}
\sup _{y \in \mathbf{Z}^{2}} & \mathbf{P}^{y}\left(\frac{L_{\sigma_{z}}(z)}{G_{n}} \leq(1-2 \delta) m_{k(n)} \ell_{n}\right) \\
\leq & e^{\lambda(1-2 \delta) m_{k(n)} \ell_{n}} \sup _{y \in \mathbf{Z}^{2}} \mathbb{E}^{y}\left(e^{-\lambda \frac{L_{\sigma_{z}}(z)}{G_{n}}}\right) \\
\leq & {\left[e^{\lambda \ell_{n}} \sup _{z^{\prime} \in \partial D_{z}\left(r_{n}\right)} \mathbb{E}^{z^{\prime}}\left(e^{-\lambda L_{T_{R_{n}}}(z) / G_{n}}\right)\right]^{(1-2 \delta) m_{k(n)}} } \\
& =\left[e^{\lambda \ell_{n}} \sup _{z \in \partial D_{0}\left(r_{n}\right)} \mathbb{E}^{z}\left(e^{-\lambda L_{T_{R_{n}}}(0) / G_{n}}\right)\right]^{(1-2 \delta) m_{k(n)}},
\end{aligned}
$$

with the last identity following from the translation invariance of SRW. We first study the quantity appearing in the first line of (8.22). By [13, Theorem 1.6.6], for all $n$ large enough,

$$
G_{R_{n}}(0,0)=\frac{2}{\pi} \log R_{n}+O(1)=\delta \frac{2}{\pi} \log n+O(1) .
$$

Hence for $n$ large enough,

$$
\begin{aligned}
(1-2 \delta) m_{k(n)} \ell_{n} G_{n} & \geq(1-2 \delta)\left(1-2 \delta^{1 / 4}\right)^{3} \frac{2}{\pi} a k^{2}(n) \\
& \geq\left(1-2 \delta^{1 / 4}\right)^{5} \frac{a}{2 \pi}(\log n)^{2}
\end{aligned}
$$

If $z \in D_{0}(\sqrt{n})$ is $n, \delta$-admissible, then necessarily

$$
L_{n}^{X, X^{\prime}}(z) \geq L_{\sigma_{z}}(z) L_{\sigma_{z}^{\prime}}^{\prime}(z)
$$

where $\sigma_{z}^{\prime}$ denotes the time it takes $\left\{X_{i}^{\prime}\right\}$ to complete $(1-2 \delta) m_{k}(n)$ excursions between $\partial D_{z}\left(r_{n}\right)$ and $\partial D_{z}\left(R_{n}\right)$, after first hitting $\partial D_{z}\left(R_{n}\right)$.

There are at most $\pi n$ lattice points $z \in D_{0}(\sqrt{n})$; hence by (8.25) and (8.24) we conclude that for $\delta \leq \delta_{0}$ and all $n$ large enough

$$
\begin{aligned}
\mathbf{P} \times & \mathbf{P}^{\prime}\left(\inf _{z \in I_{n, \delta}} L_{n}^{X, X^{\prime}}(z) \leq \frac{\left(1-2 \delta^{1 / 4}\right)^{10} a^{2}}{4 \pi^{2}}(\log n)^{4}\right) \\
& \leq 2 \pi n \sup _{y \in \mathbf{Z}^{2}} \mathbf{P}^{y}\left(L_{\sigma_{z}}(z) \leq(1-2 \delta) m_{k(n)} \ell_{n} G_{n}\right)
\end{aligned}
$$

and (8.20) will follow once we show that the right-hand side of (8.22) is bounded by $n^{-2}$, which we now do.

Let $T_{0}$ be the first hitting time of 0 and set $q_{n, z}:=\mathbf{P}^{z}\left(T_{0}<T_{R_{n}}\right)$. The Markov property followed by the inequality $e^{-t} \leq 1-t+t^{2} / 2 ; t \geq 0$ shows that

$$
\begin{aligned}
\mathbb{E}^{z}\left(e^{-\lambda L_{T_{R_{n}}}(0) / G_{n}}\right) \\
\quad=\mathbf{P}^{z}\left(T_{0}>T_{R_{n}}\right)+\mathbf{P}^{z}\left(T_{0}<T_{R_{n}}\right) \mathbb{E}^{0}\left(e^{-\lambda L_{T_{R_{n}}}(0) / G_{n}}\right) \\
\quad \leq 1-q_{n, z}+q_{n, z}\left(1-\lambda \mathbb{E}^{0}\left(\frac{L_{T_{R_{n}}}(0)}{G_{n}}\right)+\frac{\lambda^{2}}{2} \mathbb{E}^{0}\left(\left\{\frac{L_{T_{R_{n}}}(0)}{G_{n}}\right\}^{2}\right)\right)
\end{aligned}
$$


and by (8.21) this gives

$$
\mathbb{E}^{z}\left(e^{-\lambda L_{T_{R}}(0)}\right) \leq 1-q_{n, z}\left(\lambda-\lambda^{2}\right) \leq e^{-q_{n, z}\left(\lambda-\lambda^{2}\right)}
$$

By [13, Proposition 1.6.7], for all $n$ large enough, and all $z \in \partial D_{0}\left(r_{n}\right)$,

$$
q_{n, z}=\frac{\log \left(R_{n} / r_{n}\right)}{\log R_{n}}+O\left(\frac{1}{\log ^{2} R_{n}}\right) \geq(1-2 \lambda) /(\delta \log n) .
$$

Then with $q_{n}:=(1-2 \lambda) /(\delta \log n)$, we see from (8.28) and (8.29) that

$$
\sup _{z \in \partial D_{0}\left(r_{n}\right)} \mathbb{E}^{z}\left(e^{-\lambda L_{T_{R}}(0) / G_{n}}\right) \leq e^{-q_{n}\left(\lambda-\lambda^{2}\right)} .
$$

Since $\ell_{n}=(1-2 \lambda) q_{n}$, we deduce that

$$
e^{\lambda \ell_{n}} \sup _{z \in \partial D_{0}\left(r_{n}\right)} \mathbb{E}^{z}\left(e^{-\lambda L_{T_{R_{n}}}(0) / G_{n}}\right) \leq e^{-\lambda^{2} q_{n}}
$$

and for all $\delta \leq \delta_{0}(a)$ and $n$ large enough,

$$
e^{-\lambda^{2} q_{n}(1-2 \delta) m_{k(n)}} \leq e^{-a k^{2}(n) /(\lambda \log n)} \leq n^{-2} .
$$

The last two displays show that the right-hand side of $(8.22)$ is bounded by $n^{-2}$. As mentioned after (8.26), this completes the proof of (8.20).

We next turn to the proof of (8.19). It is here that we use strong approximation; so we need to introduce further notation concerning Brownian paths. Let $M_{k}^{x}$ denote the number of excursions of $\left\{W_{t}\right\}$ from $\partial D\left(x, e^{-k+1}\right)$ to $\partial D\left(x, e^{-k}\right)$ prior to $\bar{\theta}$, and let $M_{k}^{\prime x}$ denote the corresponding number of excursions for the process $\left\{W_{t}^{\prime}\right\}$. Fix $\delta, \beta>0$ and let $\mathcal{U}_{k}(\beta)$ be a fixed maximal collection of points $x_{j} \in$ $D(0,1) \cap D^{c}(0, \beta)$ with $\left|x_{i}-x_{j}\right| \geq \delta^{2} e^{-k}$ for all $x_{i}, x_{j} \in \mathcal{U}_{k}(\beta)$. We say that a point $x \in \mathcal{U}_{k}(\beta)$ is $k$-admissible if $M_{k}^{x} \wedge M_{k}^{\prime x} \geq(1-2 \delta) m_{k}$ and denote by $\widehat{\mathcal{U}}_{k}(\beta)$ the set of $k$-admissible points.

We now show how to derive (8.19) from the following lemma whose proof is momentarily deferred.

Lemma 8.4. For $\beta>0$ sufficiently small,

$$
\liminf _{k \rightarrow \infty} \mathbf{P} \times \mathbf{P}^{\prime}\left(\left|\widehat{\mathcal{U}}_{k}(\beta)\right| \geq e^{2(1-a-2 \delta) k}\right) \geq 7 / 8 .
$$

Using (8.32) together with the fact that $\bar{\theta} \vee \bar{\theta}^{\prime}<\infty$ a.s., we can find $K<\infty$ so that

$$
\liminf _{k \rightarrow \infty} \mathbf{P} \times \mathbf{P}^{\prime}\left(\left|\widehat{\mathcal{U}}_{k}(\beta)\right| \geq e^{2(1-a-2 \delta) k} ; \bar{\theta} \vee \bar{\theta}^{\prime} \leq K\right) \geq 3 / 4
$$

By Brownian scaling and the multi-dimensional strong approximation of $[6$, Theorem 1], we may construct for each $n$, independent SRW's $\left\{X_{i}\right\},\left\{X_{i}^{\prime}\right\}$ and independent Brownian motions $\left\{W_{t}: t \in[0, K]\right\},\left\{W_{t}^{\prime}: t \in[0, K]\right\}$ on the same probability space such that $\mathbf{P} \times \mathbf{P}^{\prime}\left(\mathcal{B}_{n}\right) \rightarrow 1$ as $n \rightarrow \infty$, where $\mathcal{B}_{n}$ is the set

$$
\left\{\sup _{0 \leq t \leq K}\left|W_{t}-\frac{\sqrt{2 K}}{\sqrt{n}} X_{[t n / K]}\right| \leq \delta e^{-k(n)} \sup _{0 \leq t \leq K}\left|W_{t}^{\prime}-\frac{\sqrt{2 K}}{\sqrt{n}} X_{[t n / K]}^{\prime}\right| \leq \delta e^{-k(n)}\right\} .
$$

Recall that if $\bar{\theta} \vee \bar{\theta}^{\prime} \leq K$, then the event that $x \in D(0,1)$ is $k$-admissible is measurable on $\sigma\left(W_{t}, W_{t}^{\prime}: t \leq K\right)$. Hence, for all $n$ large enough, if in addition $\mathcal{B}_{n}$ holds, then to each $k(n)$-admissible $x \in D(0,1)$ corresponds $\widetilde{x} \in \mathbf{Z}^{2}$ nearest to $\sqrt{n /(2 K)} x$ that is $n, \delta$-admissible. Since $\mathbf{P} \times \mathbf{P}^{\prime}\left(\mathcal{B}_{n}\right) \rightarrow 1$ as $n \rightarrow \infty$, (8.19) follows. 
Proof of Lemma 8.4. We begin by using the techniques of [5] to find many points in whose neighborhood both Brownian paths have large occupation measure. Let

$$
\mathcal{C}_{a}:=\left\{x \in D(0,1): \lim _{\epsilon \rightarrow 0} \frac{\mu_{\bar{\theta}}^{w}(D(x, \varepsilon))}{\varepsilon^{2}(\log \epsilon)^{2}}=a\right\},
$$

and let $\mathcal{C}_{a}^{\prime}$ denote the corresponding set for the process $\left\{W_{t}^{\prime}\right\}$.

\section{Lemma 8.5.}

$$
\mathbf{P} \times \mathbf{P}^{\prime}\left\{\operatorname{dim}\left(\mathcal{C}_{a} \cap \mathcal{C}_{a}^{\prime}\right)=2-2 a\right\}=1 .
$$

Proof of Lemma 8.5. We follow the proof of [5, Theorem 1.3], but we now say that the indicator function $Y(n, i)$ introduced in [5, Section 3] is equal to 1 iff $x_{n, i}$ is $n$-perfect both for $W$ and $W^{\prime}$. By independence, the bounds for the first moment and covariance of $Y(n, i)$ which appear in [5, Lemma 3.2] now have $a$ replaced by $2 a$. The rest of the proof now proceeds exactly as in [5].

The following lemma allows us to obtain large excursion counts from the large occupation times provided by the previous lemma. We abbreviate $\rho_{k}=e^{-k}$.

Lemma 8.6. For any $\delta, \beta, \gamma>0$ we can find $k_{0}=k_{0}(\omega)<\infty$ a.s. such that for all $k>k_{0}$ and $x \in \mathcal{U}_{k}(\beta)$, if

$$
\mu_{\bar{\theta}}^{W}\left(D\left(x, \rho_{k}\right)\right) \geq a\left(1-\gamma^{2}\right)\left|\rho_{k} \log \rho_{k}\right|^{2},
$$

then $M_{k}^{x} \geq(1-\gamma) m_{k}$.

Proof of Lemma 8.6. We will say that $x \in \mathcal{U}_{k}(\beta)$ is *-thick if it satisfies (8.36). Assuming $k$ is large enough so that $0 \notin D\left(x, \rho_{k}\right)$ for all $x \in \mathcal{U}_{k}(\beta)$, we let $\tau_{l, k}$ denote the occupation measure of $D\left(x, \rho_{k}\right)$ during the $l$ th excursion of $W$ between $\partial D\left(x, \rho_{k}\right)$ and $\partial D\left(x, \rho_{k-1}\right)$. Then, with $\mathbb{E}\left(\tau_{l, k}\right)=\rho_{k}^{2}$ and $m_{k}^{\prime}=(1-\gamma) m_{k}=$ $a(1-\gamma)\left(\log \rho_{k}\right)^{2}$, we have that for some universal constant $C<\infty$ and all $x \in \mathcal{U}_{k}(\beta)$,

$$
\begin{aligned}
\mathbf{P}\left(M_{k}^{x} \leq m_{k}^{\prime}, x \text { is }{ }^{*} \text {-thick }\right) & \leq \mathbf{P}\left(\sum_{l=1}^{m_{k}^{\prime}} \tau_{l, k} \geq a\left(1-\gamma^{2}\right)\left|\rho_{k} \log \rho_{k}\right|^{2}\right) \\
& =\mathbf{P}\left(\frac{1}{m_{k}^{\prime}} \sum_{l=1}^{m_{k}^{\prime}} \tilde{\tau}_{l, k} \geq \gamma\right) \leq e^{-\gamma^{2} m_{k} / C},
\end{aligned}
$$

where $\tilde{\tau}_{l, k}:=\tau_{l, k} / \mathbb{E}\left(\tau_{l, k}\right)-1$, and the last inequality follows by the methods used in the proof of [5. Lemma 6.4]. Consequently,

$$
\sum_{k=1}^{\infty} \sum_{x \in \mathcal{U}_{k}(\beta)} \mathbf{P}\left(M_{k}^{x} \leq m_{k}^{\prime}, x \text { is }{ }^{-} \text {-thick }\right) \leq \sum_{k=1}^{\infty}\left|\mathcal{U}_{k}(\beta)\right| e^{-\gamma^{2} m_{k} / C}<\infty .
$$

We can now complete the proof of Lemma 8.4. It follows from (8.35) that for some $\beta>0$ sufficiently small and all $k$ sufficiently large, with probability $\geq 7 / 8$ the set of $x \in D(0,1) \cap D^{c}(0,2 \beta)$ with

$$
\min \left(\frac{\mu_{\bar{\theta}}^{w}\left(D\left(x,\left(1-\delta^{2}\right) \rho_{k}\right)\right)}{\rho_{k}^{2}\left(\log \rho_{k}\right)^{2}}, \frac{\mu_{\bar{\theta}^{\prime}}^{w^{\prime}}\left(D\left(x,\left(1-\delta^{2}\right) \rho_{k}\right)\right)}{\rho_{k}^{2}\left(\log \rho_{k}\right)^{2}}\right) \geq\left(1-2 \delta^{2}\right) a
$$


has Hausdorff dimension $\geq 2-2 a-\delta$. Let $\widetilde{\mathcal{U}}_{k}$ be the set of points in $\mathcal{U}_{k}(\beta)$ that are within $\delta^{2} \rho_{k}$ of the set in (8.38). Using Lemma 8.6, it is easy to check that each point in $\widetilde{\mathcal{U}}_{k}$ is $k$-admissible. Since $\left\{D\left(x, \delta \rho_{k}\right): x \in \widetilde{\mathcal{U}}_{k}\right\}$ is a cover of the set in (8.38) by sets of maximal diameter $\delta^{2} \rho_{k}$, it follows that

$$
\liminf _{k \rightarrow \infty}\left|\widetilde{\mathcal{U}}_{k}\right|\left(\delta^{2} \rho_{k}\right)^{2-2 a-2 \delta}=\infty .
$$

Our lemma now follows.

\section{Complements And unsolved PROBlems}

- By Brownian scaling, for any deterministic $0<r<\infty$, the set $D(0,1)$ and $\bar{\theta}, \bar{\theta}^{\prime}$ can be replaced by $D(0, r)$ and $\bar{\theta}_{r}=\inf \left\{s:\left|W_{s}\right|=r\right\}, \bar{\theta}_{r}^{\prime}=\inf \left\{t:\left|W_{t}^{\prime}\right|=r\right\}$, without changing the conclusion of Theorem 1.4. Similarly, one may replace $\mathcal{I}_{\bar{\theta}}, \bar{\theta}^{\prime}$ by $\mathcal{I}_{S, T}$ in this theorem, for any deterministic $0<S, T<\infty$. Moreover, from its proof we have that (1.10) remains valid when the limit in $\varepsilon$ is replaced by liminf or limsup and when considering the set of points $x$ for which this limit (lim inf, lim sup, respectively) is at least $a^{2}$.

- Next, we discuss briefly the packing dimension analogue of Theorem 1.4 consult Mattila (1995) for background on packing dimension, Minkowski dimension and their relation. The set of consistently thick points CThicklnt $\geq_{a^{2}}$, defined in (5.2), has different packing dimension from the set Thicklnt $\geq a^{2}$, defined in (2.8). Namely, for every $a \in(0,1]$,

$$
\begin{gathered}
\operatorname{dim}_{P}(\text { CThicklnt } \\
\operatorname{dim}_{P}(\text { Thicklnt } \\
\left.\operatorname{la}^{2}\right)=2,2 a, \text { a.s. } \\
\text { a.s. }
\end{gathered}
$$

To justify (9.1), we use the notation of Section 2. The sets $\mathcal{A}_{n}$, defined in (2.13), satisfy

$$
\left|\mathcal{A}_{n}\right| \leq\left(\tilde{\epsilon}_{n}\right)^{(1-11 \delta) 2 a-2}
$$

for all large $n$, by (2.15) and Borel-Cantelli.

Recall the discs $\mathcal{V}_{n, j}=D\left(x_{j}, \delta \tilde{\epsilon}_{n}\right)$ defined after (2.15), and denote $\mathcal{V}_{n}=$ $\bigcup_{j \in \mathcal{A}_{n}} \mathcal{V}_{n, j}$. By (9.3), the upper Minkowski dimension of $\mathcal{V}_{\ell}^{*}=\bigcap_{n \geq \ell} \mathcal{V}_{n}$ is at most $2-(1-11 \delta) 2 a$. It is easy to see that CThicklnt $\geq a^{2} \subset \bigcup_{\ell>1} \mathcal{V}_{\ell}^{*}$, whence $\operatorname{dim}_{P}\left(\right.$ CThicklnt $\left.\geq a^{2}\right) \leq 2-(1-11 \delta) 2 a$. Since $\delta$ can be taken arbitrarily small, while $\operatorname{dim}_{P}\left(\right.$ CThicklnt $\left.\geq a^{2}\right) \geq \operatorname{dim}\left(\right.$ CThicklnt $\left.\geq a^{2}\right)$, this proves (9.1).

To prove (9.2), it clearly suffices to consider $a=1$. Recall that $\bar{\theta}=\inf \{t$ : $\left.\left|W_{t}\right|=1\right\}$. For each $n \geq 1$, let

$$
V_{n}:=\bigcup_{0<\varepsilon<1 / n}\left\{0<t<\bar{\theta}: \frac{\mathcal{I}_{\bar{\theta}, \bar{\theta}^{\prime}}\left(D\left(W_{t}, \varepsilon\right)\right)}{\varepsilon^{2}\left(\log \frac{1}{\varepsilon}\right)^{4}}>1-1 / n\right\} .
$$

The sets $V_{n}$ are open by the path continuity of Brownian motion. Moreover, it is easy to check, e.g., by applying Theorem 1.3 with an arbitrary $T$ replacing $\bar{\theta}$ there, and using the shift invariance of Brownian motion, that for any $n \geq 1$, almost surely $V_{n}$ is a dense subset of $(0, \bar{\theta})$; by [2, Corollary 2.4, part (i)], $\operatorname{dim}_{P}\left(\bigcap_{n} V_{n}\right)=1$ a.s. The set

$$
\left\{0 \leq t \leq \bar{\theta}: \limsup _{\varepsilon \rightarrow 0} \frac{\mathcal{I}_{\bar{\theta}, \bar{\theta}^{\prime}}\left(D\left(W_{t}, \varepsilon\right)\right)}{\varepsilon^{2}\left(\log \frac{1}{\varepsilon}\right)^{4}} \geq 1\right\}
$$


contains $\bigcap_{n} V_{n}$; so it has packing dimension 1. Finally, Thicklnt $\geq 1$ is the image under planar Brownian motion of the set in (9.4); hence the uniform doubling of packing dimension by planar Brownian motion (see [17, Corollary 5.8]) yields (9.2).

- The situation for intersections of $m$ independent planar Brownian motions is completely analogous to that of Theorems 1.3 and 1.4 Specifically, let $W_{s_{1}}^{(1)}, \ldots$, $W_{s_{m}}^{(m)}$ denote $m$ independent planar Brownian motions, and define the $m$-fold projected intersection local time by

$$
\begin{aligned}
& \mathcal{I}_{m, S_{1}, \ldots, S_{m}}(A) \\
& =\lim _{\epsilon \rightarrow 0} \pi^{m-1} \int_{0}^{S_{1}} \ldots \int_{0}^{S_{m}} 1_{A}\left(W_{s_{1}}^{(1)}\right) \prod_{j=2}^{m} f_{\epsilon}\left(W_{s_{1}}^{(1)}-W_{s_{j}}^{(j)}\right) d s_{1} \ldots d s_{m}
\end{aligned}
$$

where $f_{\epsilon}$ is any approximate $\delta$-function. It is known that the limit (1.8) exists a.s. and in all $L^{p}$ spaces, and that $\mathcal{I}_{m, S_{1}, \ldots, S_{m}}(\cdot)$ is a measure supported on

$$
\left\{x \in \mathbb{R}^{2} \mid x=W_{s_{1}}^{(1)}=\cdots=W_{s_{m}}^{(m)} \text { for some } 0 \leq s_{1} \leq S_{1}, \ldots, 0 \leq s_{m} \leq S_{m}\right\} ;
$$

see [?, Theorem 1, Chapter VIII]. Note that $\mathcal{I}_{1, \bar{\theta}}$ is the occupation measure studied in [5]. Let $\bar{\theta}^{(j)}=\inf \left\{s:\left|W_{s}^{(j)}\right|=1\right\}$. Then, for any $m \geq 1$,

$$
\lim _{\varepsilon \rightarrow 0} \sup _{x \in \mathbb{R}^{2}} \frac{\mathcal{I}_{m, \bar{\theta}^{(1)}, \ldots, \bar{\theta}^{(m)}}(D(x, \varepsilon))}{\varepsilon^{2}\left(\log \frac{1}{\varepsilon}\right)^{2 m}}=(2 / m)^{m}, \quad \text { a.s. }
$$

and for any $0<a \leq 2 / m$,

$$
\operatorname{dim}\left\{x \in D(0,1): \lim _{\varepsilon \rightarrow 0} \frac{\mathcal{I}_{m, \bar{\theta}^{(1)}, \ldots, \bar{\theta}^{(m)}}(D(x, \varepsilon))}{\varepsilon^{2}\left(\log \frac{1}{\varepsilon}\right)^{2 m}}=a^{m}\right\}=2-m a, \quad \text { a.s. },
$$

- The Hausdorff dimension of the set of thick intersection points for two independent Brownian motions in $\mathbb{R}^{3}$ was recently determined by König and Mörters. Specifically, let $B(x, r)$ denote the ball of radius $r$ centered at $x \in \mathbb{R}^{3}$ and $\mathcal{J}(B(x, r))$ the total intersection local time in $B(x, r)$ for two independent Brownian motions in $\mathbb{R}^{3}$ (see [12] Section 2.1] for various equivalent definitions of $\mathcal{J}(\cdot)$ ). In [12, Theorem 1.4], König and Mörters show that for any $0<a \leq \rho^{*} / 2$, almost surely,

$$
\operatorname{dim}\left\{x \in \mathbb{R}^{3}: \limsup _{\varepsilon \rightarrow 0} \frac{\mathcal{J}(B(x, \varepsilon))}{\varepsilon\left(\log \frac{1}{\varepsilon}\right)^{2}}=a^{2}\right\}=1-2 a / \rho^{*},
$$

where the non-random $\rho^{*}>0$ is the solution of the explicit variational formula [12] (1.9)]. The analog of (9.7) for consistently thick points, that is, with lim inf instead of lim sup, involves a different gauge function and remains an open problem.

- In [3] we analyzed "thin points" for the Brownian occupation measure, establishing that

$$
\lim _{\varepsilon \rightarrow 0} \inf _{t \in[0,1]} \frac{\mu_{\bar{\theta}}^{W}\left(D\left(W_{t}, \varepsilon\right)\right)}{\varepsilon^{2} / \log \frac{1}{\varepsilon}}=1, \quad \text { a.s. }
$$

with the multi-fractal spectrum

$$
\operatorname{dim}\left\{x \in D(0,1): \liminf _{\varepsilon \rightarrow 0} \frac{\mu_{\bar{\theta}}^{W}(D(x, \varepsilon))}{\varepsilon^{2} / \log \frac{1}{\varepsilon}}=a\right\}=2-2 / a, \quad \text { a.s. }
$$

for any fixed $a>1$. In the present paper, we analyzed Brownian projected intersection local time where it is exceptionally "thick". The analysis of the corresponding 
"thin intersection points" is needed to describe completely the multi-fractal structure of this measure, and remains an open problem.

- The strong approximation of SRW by Brownian motion is key to our analysis of intersections of two (or more) independent SRW's, leading to Theorem 1.1. The random walk question analogous to Theorem 1.6 is to study the number of thick points for intersections of two independent random walks in $\mathbf{Z}^{2}$ : a simple (symmetric) random walk and a symmetric transient random walk such that $X_{1}$ is in the domain of attraction of a stable symmetric law. Lacking strong approximation for the latter, this question remains an open problem.

\section{ACKNOWLEDGMENT}

We thank Wolfgang König and Peter Mörters for providing us with the preprint [12. We thank Olivier Daviaud for pointing out certain inaccuracies in the first draft of this work.

\section{REFERENCES}

1. R. Bass and D. Khoshnevisan, Intersection local times and Tanaka formulas, Ann. Inst. Henri Poincaré Probab. Statist. 29 (1993), 419-451. MR 95c:60073

2. A. Dembo, Y. Peres, J. Rosen and O. Zeitouni, Thick points for spatial Brownian motion: multi-fractal analysis of occupation measure, Ann. Probab. 28 (2000), 1-35. MR 2001g:60194

3. A. Dembo, Y. Peres, J. Rosen and O. Zeitouni, Thin points for Brownian Motion, Annales de L'Institut Henri Poincaré, 36 (2000), 749-774. CMP 2001:05

4. A. Dembo, Y. Peres, J. Rosen and O. Zeitouni, Thick points for transient symmetric stable processes, Electron. J. of Probab. 4 (1999) Paper no. 10, 1-13. MR 2000f:60117

5. A. Dembo, Y. Peres, J. Rosen and O. Zeitouni, Thick points for planar Brownian motion and the Erdös-Taylor conjecture on random walk, Acta Math. 186 (2001), 239-270.

6. U. Einmahl, Extensions of results of Komlós, Major, and Tusnády to the multivariate case. J. Multivariate Anal. 28 (1989), 20-68. MR 90g:60032

7. P. Fitzsimmons and J. Pitman, Kac's moment formula and the Feynman-Kac formula for additive functionals of a Markov process, Stochastic Processes and Applications. 79 (1999), 117-134. MR 2000a:60136

8. K. Ito and H. McKean, Diffusion processes and their sample paths, Springer-Verlag, NewYork, (1974). MR 49:9963

9. J.-P. Kahane, Some random series of functions: Second Edition, Cambridge University Press, (1985). MR 87m:60119

10. R. Kaufman, Une propriété metriqué du mouvement brownien, C. R. Acad. Sci. Paris 268 (1969), 727-728. MR 39:2219

11. J. F. Le Gall, The exact Hausdorff measure of Brownian multiple points, Seminar on Stochastic Processes 1986, E. Cinlar, K.L. Chung, R.K. Getoor, editors, Birkhäuser (1987), 107-137. MR 89a:60188

12. W. König and P. Mörters, Brownian intersection local times: upper tail asymptotics and thick points, Preprint (2001). To appear, Ann. Probab. (2002).

13. G. F. Lawler, Intersections of Random Walks, Birkhäuser, Boston, 1991. MR 92f:60122

14. J.-F. Le Gall, Some properties of planar Brownian motion, École d'été de probabilités de St. Flour XX, 1990 (Berlin). Lecture Notes Math, 1527, Springer-Verlag, Berlin, 1992. MR 94g:60156

15. J.-F. Le Gall, The exact Hausdorff measure of Brownian multiple points I and II, Seminar on Stochastic Processes, 1986, 107-137 and 1988, 193-197, Birkhäuser, Boston. MR 89a:60188. MR 90f:60139

16. P. Mattila, Geometry of sets and measures in Euclidean spaces, Cambridge University Press, (1995). MR 96h:28006

17. E. A. Perkins and S. J. Taylor, Uniform measure results for the image of subsets under Brownian motion, Probab. Theory Related Fields 76 (1987), 257-289. MR 88m:60122

18. P. Révész, Random Walk in Random and Non-Random Environments, World Scientific, Teaneck, 1990. MR 92c:60096 
Departments of Mathematics and Statistics, Stanford University, Stanford, CaliFORNIA 94305

E-mail address: amir@math.stanford.edu

Department of Statistics, University of California Berkeley, Berkeley, California 94720 and Institute of Mathematics, Hebrew University, Jerusalem, Israel

E-mail address: peres@stat.berkeley.edu

Department of Mathematics, College of Staten Island, CUny, Staten Island, New YORK 10314

E-mail address: jrosen3@earthlink.net

Department of Electrical Engineering, Technion, Haifa 32000, Israel

E-mail address: zeitouni@ee.technion.ac.il 